\title{
The SECQ, Linear Regularity and the Strong CHIP for Infinite System of Closed Convex Sets in Normed Linear Spaces
}

\author{
Chong Li ${ }^{*}$ K. F. Ng ${ }^{\dagger}$ and T. K. Pong ${ }^{\ddagger}$
}

\begin{abstract}
We consider a (finite or infinite) family of closed convex sets with nonempty intersection in a normed space. A property relating their epigraphs with their intersection's epigraph is studied, and its relations to other constraint qualifications (such as the linear regularity, the strong CHIP and Jameson's $(G)$-property) are established. With suitable continuity assumption we show how this property can be ensured from the corresponding property of some of its finite subfamilies.
\end{abstract}

Key words: System of closed convex sets, interior-point condition, strong conical hull intersection property.

AMS(MOS) Subject Classifications. Primary, 90C34; 90C25 Secondary, 52A05; 41A29

\section{Introduction}

In dealing with a lower semicontinuous extended real valued function $\phi$ defined on a Banach space (or more generally, a normed linear space) $X$, it is not only natural but also useful to study its relation with the epigraph epi $\phi:=\{(x, r) \in X \times \mathbb{R}: \phi(x) \leq r\}$, which is clearly a closed convex subset of the product $X \times \mathbb{R}$. Conversely, given a nonempty closed convex set $C$ in $X$, let $\sigma_{C}$ denote the support function of $C$, which is defined by

$$
\sigma_{C}\left(x^{*}\right)=\sup \left\{\left\langle x^{*}, x\right\rangle: x \in C\right\}, \quad x^{*} \in X^{*},
$$

where $X^{*}$ denotes the dual space of $X$ and $\left\langle x^{*}, x\right\rangle=x^{*}(x)$, the value of the functional $x^{*}$ at $x$. Thus $\sigma_{C}$ is a $w^{*}$-lower semicontinuous convex function and epi $\sigma_{C}$ is a $w^{*}$-closed convex subset of $X^{*} \times \mathbb{R}$. In this paper, we shall apply this simple duality between $C$ and epi $\sigma_{C}$ to study several important aspects (including the regularity, the strong CHIP, Jameson's property $(G)$ and other constraint qualifications) for a $C C S$-system $\left\{C_{i}: i \in I\right\}$ by which we mean a family of closed convex sets in $X$ with nonempty intersection $\bigcap_{i \in I} C_{i}$, where $I$ is an index set.

When $I$ is finite, the concept of regularity and its quantitative versions were introduced in $[4,5,6]$ by Bauschke and Borwein, and were utilized to establish norm or linear convergence results. The concept of strong conical hull intersection property (the strong CHIP for short) was introduced by Deutsch, Li and

\footnotetext{
*Department of Mathematics, Zhejiang University, Hangzhou 310027, P. R. China, (cli@zju.edu.cn). This author was supported in part by the National Natural Science Foundation of China (grant 10271025) and Program for New Century Excellent Talents in University.

†Department of Mathematics, Chinese University of Hong Kong, Hong Kong, P. R. China, (kfng@math.cuhk.edu.hk). This author was supported by a direct grant (CUHK) and an Earmarked Grant from the Research Grant Council of Hong Kong.

‡Department of Mathematics, Chinese University of Hong Kong, Hong Kong, P. R. China, (tkpong@math.cuhk.edu.hk).
} 
Ward in [12], and was utilized in [13] as well as in [9, 23, 24] to reformulate certain optimization problems with constraints. All the works cited above were in the Hilbert space or Euclidean space setting. The concept of property $(G)$ was introduced by Jameson [16] for a pair of cones, and was utilized to give a duality characterization of the linear regularity. In improving the partial results obtained by Lewis, Pang (see [22, 29]) and by Bauschke, Borwein and Li [6], Jameson's result was extended by $\mathrm{Ng}$ and Yang [28] to the general case (without additional assumption that each $C_{i}$ is a cone), but still only for finite $I$. For the case when $X$ is a Hilbert space, the same result was also independently obtained by Bakan, Deutsch and $\mathrm{Li}$ in [3].

In this paper, we extend the above mentioned results to cover the case when $I$ is infinite. From both the theoretical and application points of view, the extension from the finite case to the infinite one is of importance. Regarding the strong CHIP, such an extension has already been done rather successfully with many interesting applications (see, for example, [26, 27]). Our investigation is through the consideration of epigraphs, and in particular by virtue of that of a new constraint qualification defined below. Our works in this connection are inspired by the recent works of Jeyakumar and his collaborates (see $[7,17,18,19,21]$, etc), who made use of epigraphs to provide sufficient conditions to ensure the strong CHIP (for finite collection of closed convex sets), and study systems of convex inequalities. We say that a $C C S$-system $\left\{C_{i}: i \in I\right\}$ satisfies the SECQ (sum of epigraphs constraint qualification) if

$$
\text { epi } \sigma_{\bigcap_{i \in I} C_{i}}=\sum_{i \in I} \text { epi } \sigma_{C_{i}} .
$$

In section 4, we study the interrelationship between this property and other constraint qualifications, especially the linear regularity. Also, since this property is a property stronger than the strong CHIP (and the converse holds in some important cases, see Theorem 3.1), it is both natural and useful to inquire whether or not the sufficient conditions originally provided to ensure the strong CHIP can in fact ensure the SECQ. In this connection, let us recall the following results proved in [26] (see in particular Theorem 4.1 and 5.1 therein). For the remainder of this section, we assume that $I$ is a compact metric space (needless to say that if $I$ is finite, then it is compact under the discrete metric) and see the next section for definitions of the undefined terms.

Theorem 1.1. Consider the CCS-system $\left\{D, C_{i}: i \in I\right\}$. Suppose that

(a) $D$ is of finite dimension;

(b) the set-valued map $i \mapsto($ aff $D) \cap C_{i}$ is lower semicontinuous on $I$;

(c) there exist $x_{0} \in \bigcap_{i \in I} C_{i}$ and $r>0$ such that

$$
(\text { aff } D) \cap B\left(x_{0}, r\right) \subseteq C_{i} \quad \text { for each } i \in I \text {; }
$$

(d) the pair $\left\{\right.$ aff $\left.D, C_{i}\right\}$ has the strong CHIP for each $i \in I$.

Then $\left\{D, C_{i}: i \in I\right\}$ has the strong CHIP.

Theorem 1.2. Consider the CCS-system $\left\{D, C_{i}: i \in I\right\}$. Suppose that

(a) $D$ is of finite dimension $l$;

(b) the set-valued map $i \mapsto(\operatorname{aff} D) \cap C_{i}$ is lower and upper semicontinuous on $I$;

(c) for any finite subset $J$ of $I$ with number of elements $|J| \leq l$, there exist $x_{0} \in D$ and $r>0$ such that

$$
(\text { aff } D) \cap B\left(x_{0}, r\right) \subseteq C_{i} \quad \text { for each } i \in J
$$


(d) for any finite subset $J$ of $I$, the subsystem $\left\{D, C_{j}: j \in J\right\}$ has the strong CHIP.

Then $\left\{D, C_{i}: i \in I\right\}$ has the strong CHIP.

In section 5, we present the corresponding results for the SECQ and as a consequence Theorems 1.1 and 1.2 are recaptured with some significant improvements. In our Corollary 5.2, condition (c) in Theorem 1.1 can be considerably weakened to require (1.2) to hold for each $i \in J$ with some finite subsets $J$ of $I$ and to allow $r$ to depend on $J$. In our Corollary 5.3, we show that the word "and upper" in Theorem 1.2 (b) can be dropped and that (d) can be weakened to require the strong CHIP holds only for subsystems $\left\{D, C_{j}: j \in J\right\}$ with $|J|=l+1$.

\section{Notations and preliminary results}

The notations used in the present paper are standard $(c f .[8,14])$. In particular, we assume throughout the whole paper that $X$ is a normed linear space (over the real field $\mathbb{R}$ or the complex field $\mathbb{C}$ ). We use $\mathbf{B}(x, \epsilon)$ to denote the closed ball with center $x$ and radius $\epsilon$. For a set $A$ in $X$ (or in $\mathbb{R}^{n}$ ), the interior (resp. relative interior, closure, convex hull, convex cone hull, linear hull, affine hull, boundary) of $A$ is denoted by int $A$ (resp. ri $A, \bar{A}, \operatorname{co} A, \operatorname{cone} A, \operatorname{span} A$, aff $A$, bd $A$ ), and the negative polar cone $A^{\ominus}$ is the set defined by

$$
A^{\ominus}=\left\{x^{*} \in X^{*}: \operatorname{Re}\left\langle x^{*}, z\right\rangle \leq 0 \text { for all } z \in A\right\},
$$

which coincides with the polar $A^{\circ}$ of $A$ when $A$ is a cone. The normal cone of $A$ at $z_{0}$ is denoted by $N_{A}\left(z_{0}\right)$ and defined by $N_{A}\left(z_{0}\right)=\left(A-z_{0}\right)^{\ominus}$. Let $Z$ be a closed convex nonempty subset of $X$. The interior and the boundary of $A$ relative to $Z$ are respectively denoted by $\operatorname{rint}_{Z} A$ and $\operatorname{bd}_{Z} A$; they are defined to be respectively the interior and the boundary of the set aff $Z \cap A$ in the metric space aff $Z$. Thus, a point $z \in \operatorname{rint}_{Z} A$ if and only if there exists $\varepsilon>0$ such that

$$
z \in(\text { aff } Z) \cap \mathbf{B}(z, \varepsilon) \subseteq A
$$

while $z \in \operatorname{bd}_{Z} A$ if and only if $z \in$ aff $Z$ and, for any $\varepsilon>0$, (aff $\left.Z\right) \cap \mathbf{B}(z, \varepsilon)$ intersects $A$ and its complement.

For a closed subset $A$ of $X$, the indicator function $\delta_{A}$ and the support function $\sigma_{A}$ of set $A$ are respectively defined by

$$
\delta_{A}(x):=\left\{\begin{array}{cc}
0, & x \in A \\
\infty, & \text { otherwise }
\end{array}\right.
$$

and

$$
\sigma_{A}\left(x^{*}\right):=\sup _{x \in A} \operatorname{Re}\left\langle x^{*}, x\right\rangle \text { for each } x^{*} \in X^{*} .
$$

Let $f$ be a proper lower semicontinuous extended real-valued function on $X$. The domain of $f$ is denoted by $\operatorname{dom} f:=\{x \in X: f(x)<+\infty\}$. Then the subdifferential of $f$ at $x \in \operatorname{dom} f$, denoted by $\partial f(x)$, is defined by

$$
\partial f(x):=\left\{z^{*} \in X^{*}: f(x)+\operatorname{Re}\left\langle z^{*}, y-x\right\rangle \leq f(y) \quad \text { for all } y \in X\right\} .
$$

Let $f, g$ be proper functions respectively defined on $X$ and $X^{*}$. Let $f^{*}, g^{*}$ denote their conjugate functions, that is

$$
\begin{aligned}
f^{*}\left(x^{*}\right) & :=\sup \left\{\operatorname{Re}\left\langle x^{*}, x\right\rangle-f(x): x \in X\right\} \quad \text { for each } x^{*} \in X^{*}, \\
g^{*}(x) & :=\sup \left\{\operatorname{Re}\left\langle x^{*}, x\right\rangle-g\left(x^{*}\right): x^{*} \in X^{*}\right\} \quad \text { for each } x \in X .
\end{aligned}
$$


The epigraph of a function $f$ on $X$ is denoted by epi $f$ and defined by

$$
\text { epi } f:=\{(x, r) \in X \times \mathbb{R}: f(x) \leq r\} .
$$

Then, for proper lower semicontinuous extended real-valued convex functions $f_{1}$ and $f_{2}$ on $X$, we have

$$
f_{1} \leq f_{2} \Longleftrightarrow f_{1}^{*} \geq f_{2}^{*} \Longleftrightarrow \operatorname{epi} f_{1}^{*} \subseteq \text { epi } f_{1}^{*},
$$

where the forward direction of the first arrow and the second equivalence are easy to verify, while the backward direction of the first arrow is standard (cf. [34, Theorem 2.3.3]).

For closed convex sets $A, B$, the following assertions are well-known and easy to verify:

$$
\begin{gathered}
\sigma_{A}=\delta_{A}^{*}, \\
N_{A}(x)=\partial \delta_{A}(x) \quad \text { for each } x \in A, \\
\sigma_{A}\left(x^{*}\right)=\operatorname{Re}\left\langle x^{*}, x\right\rangle \Leftrightarrow x^{*} \in N_{A}(x) \Longleftrightarrow\left(x^{*}, \operatorname{Re}\left\langle x^{*}, x\right\rangle\right) \in \operatorname{epi} \sigma_{A} \quad \text { for each } x \in A
\end{gathered}
$$

and

$$
\text { epi } \sigma_{A} \subseteq \text { epi } \sigma_{B} \text { if } A \supseteq B \text {. }
$$

For simplicity of notations, we will usually assume that the scalar field of $X$ is $\mathbb{R}$ ( and so $\operatorname{Re}\left\langle x^{*}, x\right\rangle$ is to be replaced by $\left\langle x^{*}, x\right\rangle$ ).

Let $\left\{A_{i}: i \in J\right\}$ be a family of subsets of $X$. The set $\sum_{i \in J} A_{i}$ is defined by

$$
\sum_{i \in J} A_{i}= \begin{cases}\left\{\sum_{i \in J_{0}} a_{i}: a_{i} \in A_{i}, \quad J_{0} \subseteq J \text { being finite }\right\}, & \text { if } J \neq \emptyset, \\ \{0\}, & \text { if } J=\emptyset .\end{cases}
$$

Let $I$ be an arbitrary index set. The following concept of the strong CHIP plays an important role in optimization theory (see $[3,6,9,10,11,31]$ ) and is due to $[12,13]$ in the case when $I$ is finite and $[25,26]$ in the case when $I$ is infinite.

Definition 2.1. Let $\left\{C_{i}: i \in I\right\}$ be a collection of convex subsets of $X$. The collection is said to have

(a) the strong CHIP at $x \in \bigcap_{i \in I} C_{i}$ if $N_{\bigcap_{i \in I} C_{i}}(x)=\sum_{i \in I} N_{C_{i}}(x)$, that is

$$
\left(\bigcap_{i \in I} C_{i}-x\right)^{\ominus}=\sum_{i \in I}\left(C_{i}-x\right)^{\ominus}
$$

(b) the strong CHIP if it has the strong CHIP at each point of $\bigcap_{i \in I} C_{i}$;

(c) the SECQ if epi $\sigma_{\bigcap_{i \in I} C_{i}}=\sum_{i \in I}$ epi $\sigma_{C_{i}}$.

Note that $N_{\bigcap_{i \in I} C_{i}}(x) \supseteq \sum_{i \in I} N_{C_{i}}(x)$ holds automatically for $x \in \bigcap_{i \in I} C_{i}$. Hence $\left\{C_{i}: i \in I\right\}$ has the strong CHIP at $x$ if and only if

$$
N_{\bigcap_{i \in I} C_{i}}(x) \subseteq \sum_{i \in I} N_{C_{i}}(x)
$$

To establish a similar property regarding the SECQ, we first need to extend [15, part X, Theorem 2.4.4] to the setting of normed linear spaces. We recall that for an arbitrary function $f$ defined on $X^{*}$, we define $\overline{\operatorname{cof}}^{w^{*}}$ by (cf. [34, Page 63])

$$
\operatorname{epi}\left(\overline{\operatorname{co} f}^{w^{*}}\right):=\overline{\operatorname{co}(\text { epi } f)}^{w^{*}}
$$


Lemma 2.1. Let $\left\{g_{i}: i \in I\right\}$ be a family of proper convex lower semicontinuous functions on a normed linear space $X$ with $\sup _{i \in I} g_{i}\left(x_{0}\right) \leq-\alpha<+\infty$ for some $x_{0} \in X$. Then the following statements are true.

(a) $\overline{\operatorname{co}\left(\inf _{i \in I}\left(g_{i}^{*}\right)\right)} w^{*}$ is a proper function on $X^{*}$.

(b) For all $x \in X,\left(\inf _{i \in I}\left(g_{i}^{*}\right)\right)^{*}(x)=\sup _{i \in I} g_{i}(x)$.

(c) For all $y^{*} \in X^{*},\left(\sup _{i \in I} g_{i}\right)^{*}\left(y^{*}\right)=\overline{\operatorname{co}\left(\inf _{i \in I}\left(g_{i}^{*}\right)\right)}{ }^{*}\left(y^{*}\right)$.

Proof. (a) Let $h: X^{*} \mapsto \mathbb{R}$ be defined by

$$
h\left(x^{*}\right)=\left\langle x^{*}, x_{0}\right\rangle+\alpha \quad \text { for each } x^{*} \in X^{*} .
$$

Let $i \in I$. By definition we have that

$$
\left\langle x^{*}, x_{0}\right\rangle-g_{i}^{*}\left(x^{*}\right) \leq g_{i}\left(x_{0}\right) \leq-\alpha \text { for each } x^{*} \in X^{*} .
$$

This shows that each $h$ is dominated by $g_{i}^{*}$ and hence that

$$
\inf _{i \in I} g_{i}^{*}\left(x^{*}\right) \geq h\left(x^{*}\right) \quad \text { for each } x^{*} \in X^{*} .
$$

Since $h$ is a $w^{*}$-continuous and affine function, it follows from the definition of closed convex hull of a function that $\overline{\operatorname{co}\left(\inf _{i \in I}\left(g_{i}^{*}\right)\right)} w^{*}\left(x^{*}\right) \geq h\left(x^{*}\right)$ for all $x^{*} \in X^{*}$. Thus $\overline{\operatorname{co}\left(\inf _{i \in I}\left(g_{i}^{*}\right)\right)} w^{*}$ is proper.

(b) For each $x \in X$, we have

$$
\begin{aligned}
\left.\operatorname{(inf}_{i \in I}\left(g_{i}^{*}\right)\right)^{*}(x) & =\sup _{x^{*} \in X^{*}} \sup _{i \in I}\left\{\left\langle x^{*}, x\right\rangle-g_{i}^{*}\left(x^{*}\right)\right\} \\
& =\sup _{i \in I} \sup _{x^{*} \in X^{*}}\left\{\left\langle x^{*}, x\right\rangle-g_{i}^{*}\left(x^{*}\right)\right\} \\
& =\sup _{i \in I} g_{i}^{* *}(x)=\sup _{i \in I} g_{i}(x),
\end{aligned}
$$

where the last equality follows from [34, Corollary 2.3.2 and Theorem 2.3.3] as each $g_{i}$ is proper convex lower semicontinuous function.

(c) Applying the conjugations to both sides of (b), we get

$$
\left(\inf _{i \in I}\left(g_{i}^{*}\right)\right)^{* *}=\left(\sup _{i \in I} g_{i}\right)^{*} .
$$

By (a), we see that $\overline{\operatorname{co}\left(\inf _{i \in I}\left(g_{i}^{*}\right)\right)} w^{*}$ is proper. Combining this with [34, Theorem 2.3.4], we see that $\left(\inf _{i \in I} g_{i}^{*}\right)^{* *}=\overline{\operatorname{co}\left(\inf _{i \in I}\left(g_{i}^{*}\right)\right)} w^{*}$, which completes the proof.

The following lemma was stated without proof in [20, P.902]. We give a proof here for the sake of completeness (Note that the condition that " $\sup _{i \in I} g_{i}$ is proper" is needed).

Lemma 2.2. Let $\left\{g_{i}: i \in I\right\}$ be a system of proper convex lower semicontinuous functions on a normed linear space $X$ with $\sup _{i \in I} g_{i}\left(x_{0}\right)<+\infty$ for some $x_{0} \in X$. Then

$$
\operatorname{epi}\left(\sup _{i \in I} g_{i}\right)^{*}=\overline{\operatorname{co} \bigcup_{i \in I} \operatorname{epi} g_{i}^{*}} w^{*} \text {. }
$$


Proof. By part (c) of Lemma 2.1, we have

$$
\operatorname{epi}\left(\sup _{i \in I} g_{i}\right)^{*}={\overline{\operatorname{co}\left(\operatorname{epi}\left(\inf _{i \in I}\left(g_{i}^{*}\right)\right)\right)}}_{w^{*}} .
$$

We claim that

$$
\overline{\operatorname{epi}\left(\inf _{i \in I}\left(g_{i}^{*}\right)\right)} w^{w^{*}}=\bar{\bigcup}_{i \in I}^{\operatorname{epi} g_{i}^{*}} w^{*} .
$$

Granting this, we see that epi $\left.\inf _{i \in I}\left(g_{i}^{*}\right)\right)$ and $\bigcup_{i \in I}$ epi $g_{i}^{*}$ have the same $w^{*}-$ closed convex hull, that is,

$$
\overline{\operatorname{co}\left(\operatorname{epi}\left(\inf _{i \in I}\left(g_{i}^{*}\right)\right)\right)} w^{*}=\overline{\operatorname{co} \bigcup_{i \in I} \operatorname{epi} g_{i}^{*}} w^{*} .
$$

Combining this with (2.11), we arrive at (2.10). Thus it remains to prove (2.12). To do this we note first that epi $g_{i}^{*} \subseteq \operatorname{epi}\left(\inf _{i \in I} g_{i}^{*}\right)$ since $g_{i}^{*} \geq \inf _{i \in I} g_{i}^{*}$ for all $i$, and thus $\overline{\bigcup_{i \in I} \text { epi } g_{i}^{*}} w^{*} \subseteq \overline{\operatorname{epi~}\left(\inf _{i \in I} g_{i}^{*}\right)} w^{*}$.

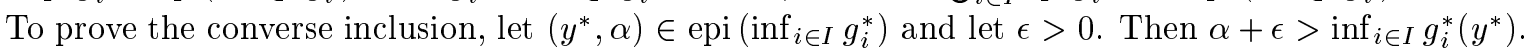
Hence there exists $i_{0} \in I$ such that $\alpha+\epsilon>g_{i_{0}}^{*}\left(y^{*}\right)$, which implies $\left(y^{*}, \alpha+\epsilon\right) \in$ epi $g_{i_{0}}^{*} \subseteq \bigcup_{i \in I}$ epi $g_{i}^{*}$. Letting $\epsilon \downarrow 0$, we get $\left(y^{*}, \alpha\right) \in \overline{\bigcup_{i \in I} \text { epi } g_{i}^{*}} w^{*}$. This proves (2.12) and thus completes the proof.

Proposition 2.1. Let $\left\{C_{i}: i \in I\right\}$ be a collection of closed convex sets in $X$ with $C:=\bigcap_{i \in I} C_{i} \neq \emptyset$. Then

$$
\text { epi } \sigma_{C}={\overline{\sum_{i \in I} \text { epi } \sigma_{C_{i}}}}^{w^{*}} \text {. }
$$

Proof. Note that $\sup _{i \in I} \delta_{C_{i}}=\delta_{C}$ and that $\sigma_{C}=\delta_{C}^{*}$ by (2.3). It follows that epi $\sigma_{C}=\operatorname{epi}\left(\sup _{i \in I} \delta_{C_{i}}\right)^{*}$. Consequently, by (2.10) and (2.3), one has that

$$
\text { epi } \sigma_{C}={\overline{\operatorname{co} \bigcup_{i \in I} \operatorname{epi} \delta_{C_{i}}^{*}}}^{w^{*}}={\overline{\operatorname{co} \bigcup_{i \in I} \operatorname{epi} \sigma_{C_{i}}}}^{w^{*}}={\overline{\sum_{i \in I} \operatorname{epi} \sigma_{C_{i}}}}^{w^{*}}
$$

where the last equality holds because epi $\sigma_{C_{i}}$ is clearly a cone for each $i \in I$.

Corollary 2.1. Let $\left\{C_{i}: i \in I\right\}$ be a collection of closed convex sets in $X$ with $C:=\bigcap_{i \in I} C_{i} \neq \emptyset$. Then the following equivalences are true:

$$
\left\{C_{i}: i \in I\right\} \text { satisfies the } S E C Q \Longleftrightarrow \sum_{i \in I} \text { epi } \sigma_{C_{i}} \text { is } w^{*}-\text { closed } \Longleftrightarrow \text { epi } \sigma_{C} \subseteq \sum_{i \in I} \text { epi } \sigma_{C_{i}} \text {. }
$$

The following simple proposition states that the SECQ is invariant under translation.

Proposition 2.2. Let $\left\{C_{i}: i \in I\right\}$ be a family of closed convex sets in $X$. Suppose that $C:=\bigcap_{i \in I} C_{i} \neq \emptyset$. Then $\left\{C_{i}: i \in I\right\}$ satisfies the SECQ if and only if the system $\left\{C_{i}-x: i \in I\right\}$ does for each $x \in X$.

Proof. Let $x \in X$. Note that

$$
\left(y^{*}, \alpha\right) \in \operatorname{epi} \sigma_{C-x} \Longleftrightarrow\left(y^{*}, \alpha+\left\langle y^{*}, x\right\rangle\right) \in \operatorname{epi} \sigma_{C}
$$

and

$$
\left(y^{*}, \alpha\right) \in \sum_{i \in I} \operatorname{epi} \sigma_{C_{i}-x} \Longleftrightarrow\left(y^{*}, \alpha+\left\langle y^{*}, x\right\rangle\right) \in \sum_{i \in I} \text { epi } \sigma_{C_{i}} .
$$

Hence the conclusion follows from Corollary 2.1. 
We will need the following notion of semicontinuity of set-valued maps in sections 4 and 5 . Readers may refer to standard texts such as [1].

Definition 2.2. Let $Q$ be a compact metric space. Let $X$ be a normed linear space and let $t_{0} \in Q$. A set-valued function $F: Q \rightarrow 2^{Y} \backslash\{\emptyset\}$ is said to be

(i) lower semicontinuous at $t_{0}$, if, for any $y_{0} \in F\left(t_{0}\right)$ and any $\epsilon>0$, there exists a neighborhood $U\left(t_{0}\right)$ of $t_{0}$ such that $\mathbf{B}\left(y_{0}, \epsilon\right) \cap F(t) \neq \emptyset$ for each $t \in U\left(t_{0}\right)$;

(ii) lower semicontinuous on $Q$ if it is lower semicontinuous at each $t \in Q$.

The following characterization regarding the lower semicontinuity is a reformulation of the equivalence of (i) and (ii) in [26, Proposition 3.1]. Let $\operatorname{lim~inf}_{t \rightarrow t_{0}} F(t)$ denote the lower limit of the set-valued function $F$ at $t_{0} \in Q$ which is defined by

$$
\liminf _{t \rightarrow t_{0}} F(t):=\left\{z \in X: \exists\left\{z_{t}\right\}_{t \in Q} \text { with } z_{t} \in F(t) \text { such that } z_{t} \rightarrow z \text { as } t \rightarrow t_{0}\right\} .
$$

Proposition 2.3. Let $Q$ be a compact metric space. Let $F: Q \rightarrow 2^{X} \backslash\{\emptyset\}$ be a set-valued function and let $t_{0} \in Q$. Then $F$ is lower semicontinuous at $t_{0}$ if and only if

$$
F\left(t_{0}\right) \subseteq \liminf _{t \rightarrow t_{0}} F(t)
$$

We collect some properties of the lower limit of the set-valued function $F$ at $t_{0} \in Q$ in the following proposition. The first property is direct from definition and the second property is a direct consequence of [30, Proposition 4.15].

Proposition 2.4. Let $Q$ be a compact metric space and $X$ a normed linear space. Let $F: Q \rightarrow 2^{X} \backslash\{\emptyset\}$ be a set-valued function such that $F(t)$ is convex for each $t \in Q$. Let $t_{0} \in Q$. Then $\liminf _{t \rightarrow t_{0}} F(t)$ is convex.

Moreover, if $X$ is finite dimensional and $B$ is a compact subset contained in $\operatorname{int}\left(\liminf _{t \rightarrow t_{0}} F(t)\right)$ (e.g. $F$ is lower semicontinuous and $B$ is a compact set contained in $\operatorname{int}\left(F\left(t_{0}\right)\right)$ ), then there exists a neighborhood $U\left(t_{0}\right)$ of $t_{0}$ such that $B \subseteq \operatorname{int} F(t)$ for each $t \in U\left(t_{0}\right)$.

\section{The strong CHIP and the SECQ}

Recall that $I$ is an arbitrary index set and $\left\{C_{i}: i \in I\right\}$ is a collection of nonempty closed convex subsets of $X$. We denote $\bigcap_{i \in I} C_{i}$ by $C$ and assume that $0 \in C$ throughout the whole paper. The following theorem describes a relationship between the strong CHIP and the SECQ for the system $\left\{C_{i}: i \in I\right\}$.

Theorem 3.1. If $\left\{C_{i}: i \in I\right\}$ satisfies the $S E C Q$, then it has the strong CHIP; the converse conclusion holds if $\operatorname{dom} \sigma_{C} \subseteq \operatorname{Im} \partial \delta_{C}$, that is if

$$
\operatorname{dom} \sigma_{C} \subseteq \bigcup_{x \in C} N_{C}(x)
$$

Proof. Suppose that $\left\{C_{i}: i \in I\right\}$ satisfies the SECQ. Let $x \in C$ and $y^{*} \in N_{C}(x)$. Then $\left(y^{*},\left\langle y^{*}, x\right\rangle\right) \in$ epi $\sigma_{C}$ by (2.5). Hence, if $\left\{C_{i}: i \in I\right\}$ satisfies the SECQ, one can apply (2.15) to express $\left(y^{*},\left\langle y^{*}, x\right\rangle\right)$ as

$$
\left(y^{*},\left\langle y^{*}, x\right\rangle\right)=\sum_{j \in J}\left(y_{j}^{*}, u_{j}\right)
$$


for some finite set $J \subseteq I$ and $\left(y_{j}^{*}, u_{j}\right) \in$ epi $\sigma_{C_{j}}(x)$ for each $j \in J$. Then $\left\langle y_{j}^{*}, x\right\rangle \leq \sigma_{C_{j}}\left(y_{j}^{*}\right) \leq u_{j}$ for all $j \in J$ and $\sum_{j \in J}\left\langle y_{j}^{*}, x\right\rangle=\sum_{j \in J} u_{j}$. It follows that $\left\langle y_{j}^{*}, x\right\rangle=u_{j}$ for each $j \in J$ and hence that $y_{j}^{*} \in N_{C_{j}}(x)$ by (2.5). Therefore $y^{*} \in \sum_{i \in I} N_{C_{i}}(x)$. Thus the strong CHIP for $\left\{C_{i}: i \in I\right\}$ is proved.

Conversely, assume that $\operatorname{dom} \sigma_{C} \subseteq \operatorname{Im} \partial \delta_{C}$ and that the strong CHIP for $\left\{C_{i}: i \in I\right\}$ is satisfied. We have to show that

$$
\text { epi } \sigma_{C} \subseteq \sum_{i \in I} \text { epi } \sigma_{C_{i}} \text {. }
$$

To do this, let $\left(y^{*}, \alpha\right) \in$ epi $\sigma_{C}$, that is, $\alpha \geq \sigma_{C}\left(y^{*}\right)$. Hence $y^{*} \in \operatorname{dom} \sigma_{C}$. Then, by the assumption and (2.5), there exists $x \in C$ such that $y^{*} \in N_{C}(x)$. By the strong CHIP assumption, it follows that there exist a finite index set $J \subseteq I$ and $y_{j}^{*} \in N_{C_{j}}(x)$ for each $j \in J$ such that

$$
y^{*}=\sum_{j \in J} y_{j}^{*} .
$$

Note that, for each $j \in J, \sigma_{C_{j}}\left(y_{j}^{*}\right) \leq\left\langle y_{j}^{*}, x\right\rangle$ because $y_{j}^{*} \in N_{C_{j}}(x)$. Since $\alpha \geq\left\langle y^{*}, x\right\rangle=\sum_{j \in J}\left\langle y_{j}^{*}, x\right\rangle$, there exists a set $\left\{\alpha_{j}: j \in J\right\}$ of real numbers such that

$$
\alpha=\sum_{j \in J} \alpha_{j} \quad \text { and } \quad \sigma_{C_{j}}\left(y_{j}^{*}\right) \leq\left\langle y_{j}^{*}, x\right\rangle \leq \alpha_{j} \quad \text { for each } j \in J .
$$

This implies that $\left(y_{j}^{*}, \alpha_{j}\right) \in$ epi $\sigma_{C_{j}}$ for each $j$ and $\left(y^{*}, \alpha\right) \in \sum_{i \in I}$ epi $\sigma_{C_{i}}$ thanks to (3.3). Hence (3.2) is proved.

Let $f$ be a proper extended real valued function on $X$ and $\bar{x} \notin \operatorname{dom} f$. Recall that the continuity of $f$ at $\bar{x}$ means that there exists a neighborhood $V$ of $\bar{x}$ such that $f(\cdot)=+\infty$ on $V$.

Proposition 3.1. Let $C$ be a nonempty closed convex set in $X$. Then the condition (3.1) holds in each of the following cases.

(i) There exists a weakly compact convex set $D$ and a closed convex cone $K$ such that $C=D+K$.

(ii) $\operatorname{dim} C<\infty, \operatorname{Im} \partial \delta_{C}$ is convex and the restriction $\left.\sigma_{C}\right|_{(\operatorname{span} C)^{*}}$ of $\sigma_{C}$ to the dual of the linear hull of $C$ is continuous.

Proof. (i). Suppose that (i) holds and let $y^{*} \in \operatorname{dom} \sigma_{C}$. Then since $K$ is a cone,

$$
\sup _{d \in D}\left\langle y^{*}, d\right\rangle=\sup _{d \in D}\left\langle y^{*}, d\right\rangle+\sup _{k \in K}\left\langle y^{*}, k\right\rangle=\sup _{d \in D, k \in K}\left\langle y^{*}, d+k\right\rangle=\sigma_{C}\left(y^{*}\right)<+\infty .
$$

Since $D$ is weakly compact, there exists $\bar{x} \in D(\subseteq C)$ such that $\left\langle y^{*}, \bar{x}\right\rangle=\sup _{d \in D}\left\langle y^{*}, d\right\rangle$. Thus by (3.5), $\left\langle y^{*}, \bar{x}\right\rangle=\sigma_{D}\left(y^{*}\right)=\sigma_{C}\left(y^{*}\right)$. Hence $y^{*} \in N_{C}(\bar{x})$ and (3.1) is proved.

(ii). Suppose that (ii) holds. If $C$ is bounded, then $C$ is compact because span $C$ is finite dimensional. Hence (3.1) in this case follows from part (i). If $C$ is the whole space, then (3.1) holds trivially as $\operatorname{dom} \sigma_{C}=\operatorname{Im} \partial \delta_{C}=\{0\}$. Thus we may assume that $C$ is a proper and unbounded subset of the finite dimensional space $Z:=$ span $C$. Let $\hat{\delta}_{C}$ and $\hat{\sigma}_{C}$ denote respectively the indicator function and the support function of the set $C$ as a set in the space $Z$. Then $\hat{\delta}_{C}$ and $\hat{\sigma}_{C}$ are respectively the restrictions onto $Z$ and $Z^{*}$ of $\delta_{C}$ and $\sigma_{C}$. It is easy to see from definitions that

$$
\operatorname{dom} \sigma_{C}=\left\{y^{*} \in X^{*}:\left.y^{*}\right|_{Z} \in \operatorname{dom} \hat{\sigma}_{C}\right\} \quad \text { and } \operatorname{Im} \partial \delta_{C}=\left\{y^{*} \in X^{*}:\left.y^{*}\right|_{Z} \in \operatorname{Im} \partial \hat{\delta}_{C}\right\} .
$$

Now, by assumption, it follows that $\operatorname{Im} \partial \hat{\delta}_{C}$ is convex in $Z^{*}$. We claim that

$$
\operatorname{dom} \hat{\sigma}_{C} \subseteq \operatorname{Im} \partial \hat{\delta}_{C}
$$


Since $C$ is proper, unbounded and the restriction $\left.\sigma_{C}\right|_{(\operatorname{span} C)^{*}}$ of $\sigma_{C}$ to the dual of the linear hull of $C$ is continuous, we know from from [2, Proposition 2.4.3] that

$$
\operatorname{dom} \hat{\sigma}_{C} \backslash\{\mathbf{0}\}=\operatorname{int}\left(\operatorname{dom} \hat{\sigma}_{\mathbf{C}}\right) \neq \emptyset .
$$

On the other hand, since $\operatorname{Im} \partial \hat{\delta}_{C}$ is a convex set in the finite dimensional Banach space $Z^{*}$, one has (cf. [34, Proposition 1.2.1 and Corollary 1.3.4])

$$
\operatorname{int}\left(\operatorname{Im} \partial \hat{\delta}_{C}\right)=\operatorname{int}\left(\overline{\operatorname{Im} \partial \hat{\delta}_{C}}\right) .
$$

Moreover, by [34, Theorem 3.1.2], one has $\operatorname{dom} \hat{\sigma}_{C} \subseteq \overline{\operatorname{Im} \partial \hat{\delta}_{C}}$. Consequently, by (3.7)-(3.9), we get that

$$
\operatorname{dom} \hat{\sigma}_{C} \backslash\{\mathbf{0}\}=\operatorname{int}\left(\operatorname{dom} \hat{\sigma}_{\mathbf{C}}\right) \subseteq \operatorname{int}\left(\overline{\operatorname{Im} \partial \hat{\delta}_{\mathbf{C}}}\right)=\operatorname{int}\left(\operatorname{Im} \partial \hat{\delta}_{\mathbf{C}}\right) \subseteq \operatorname{Im} \partial \hat{\delta}_{\mathbf{C}} .
$$

Therefore the claim (3.7) stands because $0 \in \operatorname{Im} \partial \hat{\delta}_{C}$. Consequently, (3.1) follows from (3.6), (3.7) and the Hahn-Banach Theorem. The proof is complete.

Remark 3.1. (i) By [2, Theorem 2.4.1], for a closed convex set $C$ with $\operatorname{dim} C<\infty$, the last condition in (ii) of Proposition 3.1 is satisfied if and only if there does not exist a half-line $\rho$ such that $\rho \subseteq \operatorname{bd} C$ nor exist a half-line $\rho$ in $(\operatorname{span} C) \backslash C$ such that $\inf \{\|x-y\|: x \in \rho, y \in C\}=0$.

(ii) Since $\operatorname{Im} \partial \delta_{C} \subseteq \operatorname{dom} \sigma_{C}$ holds automatically, (3.1) is equivalent to $\operatorname{Im} \partial \delta_{C}=\operatorname{dom} \sigma_{C}$. Thus, by the convexity of $\operatorname{dom} \sigma_{C}$, the convexity assumption of $\operatorname{Im} \partial \delta_{C}$ in (ii) of Proposition 2.1 is necessary for (3.1).

Combining Theorem 3.1 and Proposition 3.1, we immediately have the following corollary.

Corollary 3.1. Let $\left\{C_{i}: i \in I\right\}$ be a family of closed convex sets in $X$. Then the strong CHIP and the $S E C Q$ are equivalent for $\left\{C_{i}: i \in I\right\}$ in each of the following cases.

(i) There exists a weakly compact convex set $D$ and a closed convex cone $K$ such that $C=D+K$.

(ii) $\operatorname{dim} C<\infty, \operatorname{Im} \partial \delta_{C}$ is convex and the restriction $\left.\sigma_{C}\right|_{(\operatorname{span} C)^{*}}$ of $\sigma_{C}$ to the dual of the linear hull of $\operatorname{span} C$ is continuous..

Remark 3.2. Part (i) was known in some special cases; see [7, Proposition 4.2] for the case when I is a two point set and $D=\{0\}$, and [19] for the case when $I$ is a finite set and $D=\{0\}$.

\section{Linear regularity and the SECQ}

Let $I$ be an arbitrary index set and let $\left\{C_{i}: \quad i \in I\right\}$ be a CCS-system with $0 \in C$, where $C=\bigcap_{i \in I} C_{i}$ as before. Throughout this section, we shall use $\boldsymbol{\Sigma}^{*}$ to denote the set $\mathbf{B}^{*} \times \mathbb{R}^{+}$, where $\mathbf{B}^{*}$ is the closed unit ball of $X^{*}$ while $\mathbb{R}^{+}$consists of all nonnegative real numbers. This section is devoted to a study of the relationship between the linear regularity and the SECQ. We begin with the notion of the linear regularity for the system $\left\{C_{i}: \quad i \in I\right\}$ and two simple lemmas (the first one is easy to verify). For a closed convex set $S$ in a normed linear space $X$, let $d_{S}(\cdot)$ denote the distance function of $S$ defined by $d_{S}(x)=\inf \{\|x-y\|: y \in S\}$ for each $x \in X$.

Definition 4.1. The system $\left\{C_{i}: i \in I\right\}$ is said to be 
(i) linearly regular if there exists a constant $\gamma>0$ such that

$$
d_{C}(x) \leq \gamma \sup _{i \in I} d_{C_{i}}(x) \quad \text { for all } x \in X .
$$

(ii) boundedly linearly regular if, for each $r>0$, there exists a constant $\gamma_{r}>0$ such that

$$
d_{C}(x) \leq \gamma_{r} \sup _{i \in I} d_{C_{i}}(x) \quad \text { for all } x \in r \mathbf{B} .
$$

Lemma 4.1. Let $\gamma>0$. Then

$$
\overline{\operatorname{co} \bigcup_{i \in I}\left(\operatorname{epi} \sigma_{C_{i}} \cap \gamma \boldsymbol{\Sigma}^{*}\right)} w^{*}+\{0\} \times \mathbb{R}^{+}=\overline{\operatorname{co} \bigcup_{i \in I}\left(\operatorname{epi} \sigma_{C_{i}} \cap \gamma \boldsymbol{\Sigma}^{*}\right)}{ }^{*} \text {. }
$$

Lemma 4.2. Let $\gamma>0$ and let $f_{\gamma}:=\frac{1}{\gamma} d_{S}$. If $0 \in S$, then

$$
\text { epi } f_{\gamma}^{*}=\operatorname{epi} \sigma_{S} \cap\left(\frac{1}{\gamma} \mathbf{B}^{*} \times \mathbb{R}^{+}\right) \text {. }
$$

Proof. By conjugation computation rules (cf. [34, Theorem 2.3.1 (v) and Proposition 3.8.3 (i)]), we have for any $x^{*} \in X^{*}$,

$$
f_{\gamma}^{*}\left(x^{*}\right)=\frac{1}{\gamma} d_{S}^{*}\left(\gamma x^{*}\right)=\frac{1}{\gamma}\left(\sigma_{S}+\delta_{\mathbf{B}^{*}}\right)\left(\gamma x^{*}\right)=\frac{1}{\gamma}\left[\sigma_{S}\left(\gamma x^{*}\right)+\delta_{\frac{1}{\gamma} \mathbf{B}^{*}}\left(x^{*}\right)\right]=\sigma_{S}\left(x^{*}\right)+\delta_{\frac{1}{\gamma} \mathbf{B}^{*}}\left(x^{*}\right) .
$$

It follows that

$$
\left(x^{*}, \alpha\right) \in \text { epi } f_{\gamma}^{*} \Longleftrightarrow \sigma_{S}\left(x^{*}\right) \leq \alpha \text { and } x^{*} \in \frac{1}{\gamma} \mathbf{B}^{*} .
$$

Since $0 \in S, \sigma_{S}\left(x^{*}\right) \leq \alpha$ implies $\alpha \geq 0$. Hence (4.4) follows from (4.6).

In the next two theorems, we shall use the graph gph $f$ of a function $f$ which is defined by

$$
\operatorname{gph} f:=\{(x, f(x)) \in X \times \mathbb{R}: x \in \operatorname{dom} f\} .
$$

Clearly, gph $f \subseteq$ epi $f$ for a function $f$ on $X$.

Theorem 4.1. Let $\gamma>0$. Then the following conditions are equivalent.

(i) For all $x \in X, d_{C}(x) \leq \gamma \sup _{i \in I} d_{C_{i}}(x)$.

(ii) epi $\sigma_{C} \cap \boldsymbol{\Sigma}^{*} \subseteq \overline{\operatorname{co\bigcup } \bigcup_{i \in I}\left(\operatorname{epi} \sigma_{C_{i}} \cap \gamma \boldsymbol{\Sigma}^{*}\right)} w^{*}$.

(iii) $\operatorname{gph} \sigma_{C} \cap \boldsymbol{\Sigma}^{*} \subseteq \overline{\operatorname{co\bigcup } \bigcup_{i \in I}\left(\operatorname{epi} \sigma_{C_{i}} \cap \gamma \boldsymbol{\Sigma}^{*}\right)} w^{*}$.

Proof. By Lemma 2.2 and Lemma 4.2, one has that

$$
\operatorname{epi}\left(\sup _{i \in I} d_{C_{i}}\right)^{*}=\overline{\operatorname{co} \bigcup_{i \in I} \operatorname{epi} d_{C_{i}}^{*}} w^{*}=\overline{\operatorname{co} \bigcup_{i \in I}\left(\operatorname{epi} \sigma_{C_{i}} \cap \Sigma^{*}\right)} w^{*} .
$$

Noting that epi $\sigma_{S}$ is a cone and making use of (2.2) and Lemma 4.2, it follows that the following equivalences are valid:

$$
\begin{aligned}
& \text { (i) holds } \Longleftrightarrow\left(\frac{1}{\gamma} d_{C}\right)^{*} \geq\left(\sup _{i \in I} d_{C_{i}}\right)^{*}
\end{aligned}
$$

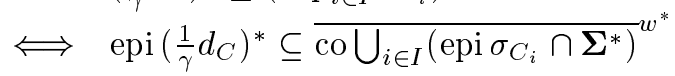

$$
\begin{aligned}
& \Longleftrightarrow \quad \text { epi } \sigma_{C} \cap \frac{1}{\gamma} \Sigma^{*} \subseteq \overline{\operatorname{co\bigcup } \bigcup_{i \in I}\left(\operatorname{epi} \sigma_{C_{i}} \cap \Sigma^{*}\right)} w^{*} \\
& \Longleftrightarrow \text { (ii) holds. }
\end{aligned}
$$


By Lemma 4.1, (iii) implies that

$$
\begin{aligned}
\text { epi } \sigma_{C} \cap \boldsymbol{\Sigma}^{*} & \subseteq \operatorname{gph} \sigma_{C} \cap \boldsymbol{\Sigma}^{*}+\{0\} \times \mathbb{R}^{+} \\
& \subseteq \overline{\operatorname{co} \bigcup_{i \in I}\left(\operatorname{epi} \sigma_{C_{i}} \cap \gamma \boldsymbol{\Sigma}^{*}\right)} w^{*}+\{0\} \times \mathbb{R}^{+} \\
& ={\overline{\operatorname{co} \bigcup_{i \in I}\left(\operatorname{epi} \sigma_{C_{i}} \cap \gamma \boldsymbol{\Sigma}^{*}\right)}}^{w^{*}} .
\end{aligned}
$$

Therefore (iii) $\Rightarrow$ (ii). Since (ii) $\Rightarrow$ (iii) is obvious, the proof is complete.

We give a simple application of our new characterization of the linear regularity in Theorem 4.1. The following theorem includes an important characterization of the linear regularity of finitely many closed convex sets in a Banach space, given in [28, Theorem 4.2].

Theorem 4.2. Let $\gamma>0$ and suppose that $X$ is a Banach space. Consider the following statements.

(i) For all $x \in X, d_{C}(x) \leq \gamma \sup _{i \in I} d_{C_{i}}(x)$.

(ii) For all $x \in C, N_{C}(x) \cap \mathbf{B}^{*} \subseteq \overline{\operatorname{co\bigcup }_{i \in I}\left(N_{C_{i}}(x) \cap \gamma \mathbf{B}^{*}\right)} w^{*}$.

Then (ii) implies (i). If assume further that $I$ is a compact metric space and $i \mapsto C_{i}$ is lower semicontinuous, then (i) and (ii) are equivalent. In particular, when I is finite, (i), (ii), (价) and (iii) are

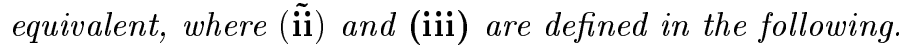

(ii) For all $x \in C, N_{C}(x) \cap \mathbf{B}^{*} \subseteq \operatorname{co\bigcup } \bigcup_{i \in I}\left(N_{C_{i}}(x) \cap \gamma \mathbf{B}^{*}\right)$.

(iii) For all $x \in C$ and for all $x^{*} \in N_{C}(x)$, there exist $x_{i}^{*} \in N_{C_{i}}(x), i \in I$ such that $\sum_{i \in I}\left\|x_{i}^{*}\right\| \leq \gamma$ and $x^{*}=\sum_{i \in I} x_{i}^{*}$.

Remark 4.1. Let $\rho>0$ and recall that the collection $\left\{D_{1}, \cdots, D_{m}\right\}$ in $X$ is said to have property $\left(G_{\rho}\right)$ if

$$
\left(\sum_{i=1}^{m} D_{i}\right) \bigcap \mathbf{B} \subseteq \sum_{i=1}^{m}\left(D_{i} \bigcap \frac{1}{\rho} \mathbf{B}\right) .
$$

Clearly, there exists $\gamma>0$ such that condition (iii) above holds if and only if the strong CHIP holds for all $x \in C$ and that there exists $\rho>0$ such that, for each $x \in C,\left\{N_{C_{i}}(x): i \in I\right\}$ has the property $\left(G_{\rho}\right)$ in $X^{*}$.

Proof. (ii) $\Rightarrow(\mathbf{i})$. Let $\epsilon>0$. In view of Theorem 4.1, to establish (i), it is sufficient to show that

$$
\operatorname{gph} \sigma_{C} \cap \boldsymbol{\Sigma}^{*} \subseteq \overline{\operatorname{co} \bigcup_{i \in I}\left(\operatorname{epi} \sigma_{C_{i}} \cap(1+\epsilon) \gamma \boldsymbol{\Sigma}^{*}\right)} w^{*} .
$$

To do this, let $\left(y^{*}, \sigma_{C}\left(y^{*}\right)\right) \in \operatorname{gph} \sigma_{C} \cap \boldsymbol{\Sigma}^{*}$. We have to show that

$$
\left(y^{*}, \sigma_{C}\left(y^{*}\right)\right) \in{\overline{\operatorname{co}} \bigcup_{i \in I}\left(\operatorname{epi} \sigma_{C_{i}} \cap(1+\epsilon) \gamma \Sigma^{*}\right)}^{w^{*}} .
$$

Consider first the case when $y^{*} \in \operatorname{Im} \partial \delta_{C}$. Then $y^{*} \in N_{C}(x) \cap \mathbf{B}^{*}$ by (2.4). Thus one can apply (ii) to find a net $\left\{\tilde{y}_{V}^{*}\right\}$ with $w^{*}$-limit $y^{*}$ such that for each $V, \tilde{y}_{V}^{*}$ is representable as

$$
\tilde{y}_{V}^{*}=\gamma \sum_{i \in J_{V}} \lambda_{i} y_{i}^{*}
$$


for some finite index set $J_{V} \subseteq I, y_{i}^{*} \in N_{C_{i}}(x) \cap \mathbf{B}^{*}, i \in J_{V}$ and $\lambda_{i} \in[0,1]$ with $\sum_{i \in J_{V}} \lambda_{i}=1$. Using (2.5) again, we obtain $\left(y_{i}^{*},\left\langle y_{i}^{*}, x\right\rangle\right) \in \operatorname{epi} \sigma_{C_{i}}$ for each $i \in J_{V}$. In $w^{*}$-limits, it follows that

$$
\left(y^{*},\left\langle y^{*}, x\right\rangle\right)=\lim _{V}\left(\tilde{y}_{V}^{*},\left\langle\tilde{y}_{V}^{*}, x\right\rangle\right)=\lim _{V} \gamma \sum_{i \in J_{V}} \lambda_{i}\left(y_{i}^{*},\left\langle y_{i}^{*}, x\right\rangle\right)
$$

hence,

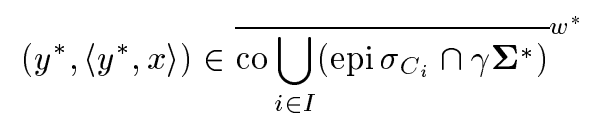

and, in particular, (4.9) holds provided that $y^{*} \in \operatorname{Im} \partial \delta_{C}$. For the general case (that is, we do not assume that $\left.y^{*} \in \operatorname{Im} \partial \delta_{C}\right)$, by [34, Theorem 3.1.4 (ii)], there exists a sequence $\left(y_{n}, y_{n}^{*}\right) \in \operatorname{gph} \partial \delta_{C}$ such that $y_{n}^{*}$ converges to $y^{*}$ in norm and $\sigma_{C}\left(y_{n}^{*}\right)$ converges to $\sigma_{C}\left(y^{*}\right)$. Note that by (2.5), we have $\left(y_{n}^{*},\left\langle y_{n}^{*}, y_{n}\right\rangle\right) \in \operatorname{gph} \sigma_{C}$. Since $\left\|y^{*}\right\| \leq 1,\left\|y_{n}^{*}\right\| \leq 1+\epsilon$ for all large enough $n$. For all such $n$ one can apply (4.11) to $\left(\frac{y_{n}^{*}}{1+\epsilon}, \sigma_{C}\left(\frac{y_{n}^{*}}{1+\epsilon}\right)\right)$ in place of $\left(y^{*}, \sigma_{C}\left(y^{*}\right)\right)$ to conclude that

$$
\left(y_{n}^{*}, \sigma_{C}\left(y_{n}^{*}\right)\right) \in \overline{\operatorname{co} \bigcup_{i \in I}\left(\operatorname{epi} \sigma_{C_{i}} \cap(1+\epsilon) \gamma \Sigma^{*}\right)}{ }^{*} .
$$

Taking limits, we get (4.9) as required. For the converse implication, let us begin to show that if (i) holds, then

$$
\partial d_{C}(x) \subseteq \gamma \partial \sup _{i \in I} d_{C_{i}}(x) \quad \text { for each } x \in C .
$$

To see this, fix any $x \in C$ and take $y^{*} \in \partial d_{C}(x)$. Then since $d_{C}(x)=0$, we obtain

$$
\left\langle y^{*}, y-x\right\rangle \leq d_{C}(y) \quad \text { for each } y \in X .
$$

Combining this with condition (i), we get

$$
\left\langle y^{*}, y-x\right\rangle \leq \gamma \sup _{i \in I} d_{C_{i}}(y) \quad \text { for each } y \in X .
$$

This implies that $y^{*} \in \gamma \partial\left(\sup _{i \in I} d_{C_{i}}\right)(x)$ and proves our claim.

Now we suppose further that $I$ is compact and that $i \mapsto C_{i}$ is lower semicontinuous. Then by [1, Corollary 1.4.17], $i \mapsto d_{C_{i}}(\cdot)$ is upper semicontinuous. Hence one can apply [34, Theorem 2.4.18] to get the inclusion $\partial\left(\sup _{i \in I} d_{C_{i}}\right)(x) \subseteq \overline{\operatorname{co\bigcup } \bigcup_{i \in I} \partial d_{C_{i}}(x)} w^{*}$, and it follows from (4.13) that $\partial d_{C}(x) \subseteq$

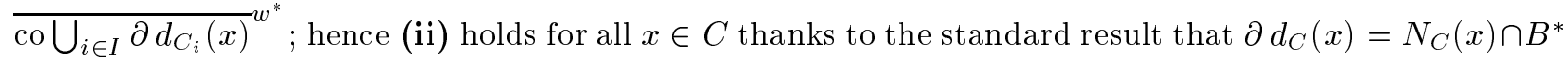
and $\partial d_{C_{i}}(x)=N_{C_{i}}(x) \cap B^{*}$ (cf. [34, Proposition 3.8.3]).

Next, we consider the case when $I$ is finite. We only need to show that (ii) $\Leftrightarrow(\tilde{\mathbf{i i}})$ in this case. For any $x \in C$, we note that by Banach-Alaoglu Theorem, $N_{C_{i}}(x) \cap \mathbf{B}^{*}$ is $w^{*}$-compact for each $i \in I$, thus co $\bigcup_{i \in I}\left(N_{C_{i}}(x) \cap \mathbf{B}^{*}\right)$ is $w^{*}$-closed as $I$ is finite. Hence (ii) and ( $\left.\tilde{\mathbf{i i}}\right)$ are the same when $I$ is finite.

Finally, we turn to prove that $(\tilde{\mathbf{i i}}) \Leftrightarrow(\mathbf{i i i})$. The forward implication is obvious. For the converse implication, fix $x \in C$. Let $x^{*} \in N_{C}(x) \cap \mathbf{B}^{*}$, we wish to show that $x^{*} \in \operatorname{co} \bigcup_{i \in I}\left(N_{C_{i}}(x) \cap \mathbf{B}^{*}\right)$. By (iii), there exist $x_{i}^{*} \in N_{C_{i}}(x), i \in I$ with $\sum_{i \in I}\left\|x_{i}^{*}\right\| \leq \gamma$ and $x^{*}=\sum_{i \in I} x_{i}^{*}$. If all the $x_{i}^{*}$ are zero, then the inclusion holds trivially. Otherwise, set $\lambda:=\sum_{i \in I}\left\|x_{i}^{*}\right\|>0$. Then $\lambda \leq \gamma$. Thus we see that

$$
x^{*}=\lambda\left(\sum_{i \in I, x_{i}^{*} \neq 0} \frac{\left\|x_{i}^{*}\right\|}{\lambda} \frac{x_{i}^{*}}{\left\|x_{i}^{*}\right\|}+\left(1-\sum_{i \in I, x_{i}^{*} \neq 0} \frac{\left\|x_{i}^{*}\right\|}{\lambda}\right) 0\right) \in \operatorname{co} \bigcup_{i \in I}\left(N_{C_{i}}(x) \cap \gamma \mathbf{B}^{*}\right),
$$

which completes the proof. 
Theorem 4.3. Suppose that

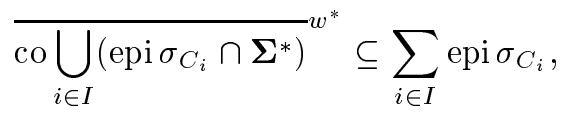

and that $\left\{C_{i}: i \in I\right\}$ is linearly regular. Then it satisfies the SECQ.

Proof. By the assumption, one can combine (4.14) with Theorem 4.1 to conclude that epi $\sigma_{C} \cap \Sigma^{*} \subseteq$ $\sum_{i \in I}$ epi $\sigma_{C_{i}}$, and hence that epi $\sigma_{C} \subseteq \sum_{i \in I}$ epi $\sigma_{C_{i}}$ for each epi $\sigma_{C_{i}}$ is a cone.

Remark 4.2. The following example shows that (4.14) in Theorem 4.3 cannot be dropped.

Example 4.1. Let $X=\mathbb{R}^{2}$ and $I=\mathbb{N}$. Define $C_{i}:=\left\{x \in X:\|x\| \leq \frac{1}{i}\right\}$ for each $i \in I$. Then $C=\bigcap_{i \in I} C_{i}=\{0\}$ and $d_{C_{i}}(x)=\max \left\{0,\|x\|-\frac{1}{i}\right\}$ for each $x \in X$. It follows that

$$
\sup _{i \in I} d_{C_{i}}(x)=\|x\|=d(x, 0)=d_{\bigcap_{i \in I} C_{i}}(x) .
$$

Hence the system $\left\{C_{i}: i \in I\right\}$ is linearly regular. On the other hand, since $C=\{0\}$ and $N_{C_{i}}(0)=\{0\}$ for each $i \in I$, this system does not have the strong CHIP. Consequently, it does not satisfy the SECQ.

In the next theorem, we shall provide some sufficient conditions for (4.14). We first prove a simple lemma. Recall that $\left\{C_{i}: i \in I\right\}$ is a CCS-system with $0 \in C$. We assume in the remainder of this section that $I$ is a compact metric space.

Lemma 4.3. Suppose that $i \mapsto C_{i}$ is lower semicontinuous. Consider elements $i_{0} \in I,\left(x_{0}^{*}, \alpha_{0}\right) \in X^{*} \times \mathbb{R}$ and nets $\left\{i_{k}\right\} \subseteq I,\left\{\left(x_{k}^{*}, \alpha_{k}\right)\right\} \subseteq X^{*} \times \mathbb{R}$ with each $\left(x_{k}^{*}, \alpha_{k}\right) \in$ epi $\sigma_{C_{i_{k}}}$. Suppose further that $i_{k} \rightarrow i_{0}$, $\alpha_{k} \rightarrow \alpha_{0}$, and that $x_{k}^{*} \rightarrow^{w^{*}} x_{0}^{*}$. If $\left\{x_{k}^{*}\right\}$ is bounded, then $\left(x_{0}^{*}, \alpha_{0}\right) \in \operatorname{epi} \sigma_{C_{i_{0}}}$.

Proof. Let $x \in C_{i_{0}}$. We have to prove that $\left\langle x_{0}^{*}, x\right\rangle \leq \alpha_{0}$. By the assumption, there exists a net $\left\{x_{k}\right\} \subseteq X$ with each $x_{k} \in C_{i_{k}}$ such that $x_{k} \rightarrow x$. Since

$$
\left\langle x_{0}^{*}, x\right\rangle=\left\langle x_{0}^{*}-x_{k}^{*}, x\right\rangle+\left\langle x_{k}^{*}, x-x_{k}\right\rangle+\left\langle x_{k}^{*}, x_{k}\right\rangle,
$$

where on the right-hand side the first two terms converge to zero and the last term $\left\langle x_{k}^{*}, x_{k}\right\rangle \leq \alpha_{k}$ for each $k$, it follows by passing to the limits that $\left\langle x_{0}^{*}, x\right\rangle \leq \alpha_{0}$.

Theorem 4.4. Suppose that $i \mapsto C_{i}$ is lower semicontinuous on $I$ and that either $I$ is finite or there exists an index $i_{0} \in I$ such that $\operatorname{dim} C_{i_{0}}<+\infty$. Then (4.14) holds. Consequently, if $\left\{C_{i}: i \in I\right\}$ is, in addition, linearly regular, then it satisfies the SECQ.

Proof. We first assume that $I$ is finite, say $I=\{1,2, \cdots, m\}$. Let $\left(\bar{x}^{*}, \bar{\alpha}\right) \in \overline{\operatorname{co\bigcup } \bigcup_{i=1}^{m}\left(\operatorname{epi} \sigma_{C_{i}} \cap \Sigma^{*}\right)} w^{*}$. Then there exists a net $\left\{\left(\bar{x}_{k}^{*}, \bar{\alpha}_{k}\right)\right\}$ in $\operatorname{co} \bigcup_{i=1}^{m}\left(\operatorname{epi} \sigma_{C_{i}} \cap \Sigma^{*}\right)$ such that $\left(\bar{x}_{k}^{*}, \bar{\alpha}_{k}\right) w^{*}$-converges to $\left(\bar{x}^{*}, \bar{\alpha}\right)$. Without loss of generality, we assume that $0 \leq \bar{\alpha}_{k} \leq \bar{\alpha}+1$ for all $k$. Each $\left(\bar{x}_{k}^{*}, \bar{\alpha}_{k}\right)$ can be expressed as a convex combination

$$
\left(\bar{x}_{k}^{*}, \bar{\alpha}_{k}\right)=\sum_{i=1}^{m} \lambda_{k, i}\left(x_{k, i}^{*}, \alpha_{k, i}\right),
$$

for some $\left(x_{k, i}^{*}, \alpha_{k, i}\right) \in$ epi $\sigma_{C_{i}} \cap \Sigma^{*}$ and $\lambda_{k, i} \in[0,1]$ with $\sum_{i=1}^{m} \lambda_{k, i}=1$. Note that

$$
\lambda_{k, i}\left(x_{k, i}^{*}, \alpha_{k, i}\right) \in \operatorname{epi} \sigma_{C_{i}} \cap \Sigma^{*} \text { for each } k \text { and } i .
$$


By considering subnets if necessary and by the $w^{*}$-compactness of the closed unit ball in Banach dual space $X^{*}$ (the Banach-Alaoglu Theorem), we may assume without loss of generality that for each $i$, there exist $x_{i}^{*} \in \mathbf{B}^{*}$ and $\beta_{i} \in[0, \bar{\alpha}+1]$ such that

$$
\lambda_{k, i} x_{k, i}^{*} \rightarrow x_{i}^{*}, \lambda_{k, i} \alpha_{k, i} \rightarrow \beta_{i}
$$

(note that $\lambda_{k, i} \alpha_{k, i} \leq \bar{\alpha}+1$ for all $k$ ). By the $w^{*}$-closedness of the set epi $\sigma_{C_{i}}$, we have from (4.17) and (4.16) that

$$
\left(x_{i}^{*}, \beta_{i}\right) \in \text { epi } \sigma_{C_{i}} \text { for each } i .
$$

Passing to limits in (4.15), we arrive at

$$
\left(\bar{x}^{*}, \bar{\alpha}\right)=\sum_{i=1}^{m}\left(x_{i}^{*}, \beta_{i}\right) \in \sum_{i=1}^{m} \operatorname{epi} \sigma_{C_{i}},
$$

where the inclusion follows from (4.18).

Next we assume that there exists an index $i_{0} \in I$ such that $\operatorname{dim} C_{i_{0}}<+\infty$. Let $Z=\operatorname{span} C_{i_{0}}$ and let $\left(\bar{x}^{*}, \bar{\alpha}\right) \in \overline{\operatorname{co} \bigcup_{i \in I}\left(\operatorname{epi} \sigma_{C_{i}} \cap \Sigma^{*}\right)} w^{*}$. Then there exists a net $\left\{\left(\bar{x}_{k}^{*}, \bar{\alpha}_{k}\right)\right\}$ in co $\bigcup_{i \in I}\left(\right.$ epi $\left.\sigma_{C_{i}} \cap \boldsymbol{\Sigma}^{*}\right)$ such that $\left(\bar{x}_{k}^{*}, \bar{\alpha}_{k}\right) \rightarrow^{w^{*}}\left(\bar{x}^{*}, \bar{\alpha}\right)$. Since $Z \times \mathbb{R}$ is of dimension $m+1$, one can apply the Caratheodory Theorem to express each $\left(\bar{x}_{k}^{*}, \bar{\alpha}_{k}\right)$ as a convex combination of $m+2$ many elements of $\bigcup_{i \in I}\left(\right.$ epi $\left.\sigma_{C_{i}} \cap \Sigma^{*}\right)$ on $Z \times \mathbb{R}$. Hence there exist indices $i_{j}^{k} \in I$, nonnegative scalars $\lambda_{k, j}$ and pairs

$$
\left(x_{k, j}^{*}, \alpha_{k, j}\right) \in \operatorname{epi} \sigma_{C_{j}^{k}} \cap \Sigma^{*} \text { for each } 1 \leq j \leq m+2
$$

with the properties $\sum_{j=1}^{m+2} \lambda_{k, j}=1$ and

$$
\left(\left.\bar{x}_{k}^{*}\right|_{Z}, \bar{\alpha}_{k}\right)=\sum_{j=1}^{m+2} \lambda_{k, j}\left(\left.x_{k, j}^{*}\right|_{Z}, \alpha_{k, j}\right) .
$$

Note that

$$
\lambda_{k, j}\left(x_{k, j}^{*}, \alpha_{k, j}\right) \in \operatorname{epi} \sigma_{C_{i k}^{k}} \cap \Sigma^{*} .
$$

Since $\left\{\bar{\alpha}_{k}\right\}$ is convergent, by passing to subnets if necessary, we may assume that $\bar{\alpha}+1 \geq \bar{\alpha}_{k} \geq 0$. Then we also have $\left\{\bar{\alpha}_{k}\right\}$ and $\left\{\lambda_{k, j} \alpha_{k, j}\right\}$ bounded for $1 \leq j \leq m+2$. Hence, considering subnets if necessary, we may assume that each of the nets $\left\{\lambda_{k, j} x_{k, j}^{*}\right\},\left\{\bar{\alpha}_{k}\right\},\left\{\lambda_{k, j} \alpha_{k, j}\right\}$ for $1 \leq j \leq m+2$ converges, say with limits,

$$
x_{0, j}^{*}, \quad \bar{\alpha}, \quad \alpha_{0, j}
$$

and we can assume further that $i_{j}^{k}$ converges to some $i_{j}^{0} \in I(1 \leq j \leq m+2)$. Making use of (4.21), it follows from Lemma 4.3 that

$$
\left(x_{0, j}^{*}, \alpha_{0, j}\right) \in \text { epi } \sigma_{C_{i_{j}^{0}}} \text { for each } 1 \leq j \leq m+2 .
$$

Moreover, passing to the limits in (4.20), we have

$$
\left(\left.\bar{x}^{*}\right|_{Z}, \bar{\alpha}\right)=\sum_{j=1}^{m+2}\left(\left.x_{0, j}^{*}\right|_{Z}, \alpha_{0, j}\right) .
$$

Noting the trivial relations that epi $\sigma_{C_{i_{0}}}$ contains $Z^{\perp} \times \mathbb{R}^{+}$, where $Z^{\perp}:=\left\{x^{*} \in X^{*}: x^{*}{ }_{Z}=0\right\}$, it follows that

$$
\left(\bar{x}^{*}, \bar{\alpha}\right) \in \sum_{j=1}^{m+2}\left(x_{0, j}^{*}, \alpha_{0, j}\right)+Z^{\perp} \times \mathbb{R}^{+} \subseteq \sum_{i \in I} \operatorname{epi} \sigma_{C_{i}} .
$$


This shows that (4.14) holds.

Finally, we, in addition, assume that $\left\{C_{i}: i \in I\right\}$ is linearly regular. Then it follows from Theorem 4.3 that this system satisfies the SECQ.

We intend to relate bounded linear regularity with the strong CHIP. We first provide a sufficient condition for a system to be linearly regular. The result is known when the ambient space is a Hilbert space ([4, Theorem 4.2.6, Corollary 4.4.4]), or a Banach space ([32, Corollary 5]). The corresponding theorems in those references are derived from a lemma whose proof is based on the open mapping theorem, and thus does not work in general normed linear spaces. As some preparation work, we first prove the following lemma, which is a generalization of [7, Proposition 3.1 (i)] to a normed linear space setting. The proof given in [7, Proposition 3.1 (i)] was based on a result in [33], while the proof we give here is a direct check on the validity of the set inclusion in (2.15).

Lemma 4.4. Let $E, F$ be two closed convex sets in $X$ with $E \cap \operatorname{int} F \neq \emptyset$. Then $\{E, F\}$ satisfies the $S E C Q$.

Proof. By Proposition 2.2, we may assume without loss of generality that $0 \in E \cap \operatorname{int} F$ and that $r \mathbf{B} \subseteq F$ for some $r>0$. Let $\left(\bar{x}^{*}, \bar{\alpha}\right) \in$ epi $\sigma_{E \cap F}$. By (2.14), there exists a $w^{*}$-convergent net $\left(x_{k}^{*}, \alpha_{k}\right)$ with limit $\left(\bar{x}^{*}, \bar{\alpha}\right)$, and for each $k,\left(x_{k}^{*}, \alpha_{k}\right) \in$ epi $\sigma_{E}+\operatorname{epi} \sigma_{F}$. Without loss of generality, we assume that $0 \leq \alpha_{k} \leq \bar{\alpha}+1$ for all $k$. Each $\left(x_{k}^{*}, \alpha_{k}\right)$ can be expressed as

$$
\left(x_{k}^{*}, \alpha_{k}\right)=\left(x_{k, 1}^{*}, \alpha_{k, 1}\right)+\left(x_{k, 2}^{*}, \alpha_{k, 2}\right),
$$

for some $\left(x_{k, 1}^{*}, \alpha_{k, 1}\right) \in \operatorname{epi} \sigma_{E}$ and $\left(x_{k, 2}^{*}, \alpha_{k, 2}\right) \in$ epi $\sigma_{F}$. Since $0 \in E$, one has $\alpha_{1} \geq \sigma_{E}\left(x_{k, 1}^{*}\right) \geq 0$ and so $\alpha_{k, 2} \leq \alpha_{k}$ for each $k$. It follows that

$$
r\left\|x_{k, 2}^{*}\right\|=\sigma_{r \mathbf{B}}\left(x_{k, 2}^{*}\right) \leq \sigma_{F}\left(x_{k, 2}^{*}\right) \leq \alpha_{k, 2} \leq \alpha_{k} \leq \bar{\alpha}+1,
$$

where the first inequality holds because $r \mathbf{B} \subseteq F$; hence $\left\{x_{k, 2}^{*}\right\}$ is a bounded net. Note also that $\alpha_{k, 1}, \alpha_{k, 2} \in$ $[0, \bar{\alpha}+1]$ as $0 \in E \cap F$ for each $k$. By considering subnets if necessary and by the $w^{*}$-compactness of the closed unit ball in Banach dual space $X^{*}$ (the Banach-Alaoglu Theorem), we may assume without loss of generality that there exist $u^{*}, v^{*}$ and $\beta_{1}, \beta_{2} \in[0, \bar{\alpha}+1]$ such that

$$
x_{k, 2}^{*} \rightarrow v^{*}, \quad x_{k, 1}^{*}=x_{k}^{*}-x_{k, 2}^{*} \rightarrow u^{*}, \quad \text { and } \quad \alpha_{k, i} \rightarrow \beta_{i} \quad \text { for each } i=1,2 .
$$

By the $w^{*}$-closedness of the epigraphs of support functions, we have from (4.23) that

$$
\left(u^{*}, \beta_{1}\right) \in \operatorname{epi} \sigma_{E} \text { and }\left(v^{*}, \beta_{2}\right) \in \operatorname{epi} \sigma_{F} .
$$

Passing to limits in (4.22), we arrive at

$$
\left(\bar{x}^{*}, \bar{\alpha}\right)=\left(u^{*}, \beta_{1}\right)+\left(v^{*}, \beta_{2}\right) \in \operatorname{epi} \sigma_{E}+\operatorname{epi} \sigma_{F} .
$$

This proves epi $\sigma_{E \cap F} \subseteq$ epi $\sigma_{E}+\operatorname{epi} \sigma_{F}$, and hence the desired result follows from Corollary 2.1.

We now give a sufficient condition for a system to be linearly regular.

Lemma 4.5. Let $E$ be a closed convex set in $X$ containing the origin and let $r>0$. Then

$$
d_{E \cap r \mathbf{B}}(x) \leq 4 \max \left\{d_{E}(x), d_{r \mathbf{B}}(x)\right\} \quad \text { for each } x \in X .
$$


Proof. We first show that

$$
\operatorname{gph} \sigma_{E \cap r \mathbf{B}} \cap \boldsymbol{\Sigma}^{*} \subseteq \operatorname{co}\left(\left(\text { epi } \sigma_{E} \cap 4 \boldsymbol{\Sigma}^{*}\right) \cup\left(\text { epi } \sigma_{r \mathbf{B}} \cap 4 \boldsymbol{\Sigma}^{*}\right)\right) .
$$

Take $\left(y^{*}, \sigma_{E \cap r \mathbf{B}}\left(y^{*}\right)\right) \in \operatorname{gph} \sigma_{E \cap r \mathbf{B}} \cap \boldsymbol{\Sigma}^{*}$. By Lemma 4.4, there exist $\left(y_{1}^{*}, \alpha_{1}\right) \in \operatorname{epi} \sigma_{E}$ and $\left(y_{2}^{*}, \alpha_{2}\right) \in$ epi $\sigma_{r \mathbf{B}}$ such that

$$
\left(y^{*}, \sigma_{E \cap r \mathbf{B}}\left(y^{*}\right)\right)=\left(y_{1}^{*}, \alpha_{1}\right)+\left(y_{2}^{*}, \alpha_{2}\right) .
$$

This implies that

$$
\sigma_{E \cap r \mathbf{B}}\left(y^{*}\right)=\alpha_{1}+\alpha_{2} .
$$

Since $0 \in E, 0 \leq \sigma_{E}\left(y_{1}^{*}\right) \leq \alpha_{1}$ and hence $\alpha_{2} \leq \sigma_{E \cap r \mathbf{B}}\left(y^{*}\right) \leq r$ thanks to (4.27). It follows that $r\left\|y_{2}^{*}\right\|=\sigma_{r \mathbf{B}}\left(y_{2}^{*}\right) \leq \alpha_{2} \leq r$, and thus $\left\|y_{1}^{*}\right\| \leq\left\|y^{*}\right\|+\left\|y_{2}^{*}\right\| \leq 2$. Therefore, that

$$
\left(y^{*}, \sigma_{E \cap r \mathbf{B}}\left(y^{*}\right)\right)=\frac{1}{2}\left[\left(2 y_{1}^{*}, 2 \alpha_{1}\right)+\left(2 y_{2}^{*}, 2 \alpha_{2}\right)\right] \in \operatorname{co}\left(\operatorname{epi} \sigma_{E} \cap 4 \boldsymbol{\Sigma}^{*} \cup \text { epi } \sigma_{r \mathbf{B}} \cap 4 \boldsymbol{\Sigma}^{*}\right),
$$

and (4.26) is established. By the implication (iii) $\Rightarrow$ (i) of Theorem 4.1 (with $\gamma=4$ ), it follows that (4.25) holds.

The following proposition on a relationship between bounded linear regularity and the linear regularity is known in [4, Theorem 4.2.6 (ii)] for the special case when $X$ is a Hilbert space.

Proposition 4.1. Let $\left\{A_{i}: i \in I\right\}$ be a system of closed convex sets in $X$ containing the origin and suppose that $\left\{A_{i}: i \in I\right\}$ is boundedly linearly regular. Then for all $r>0$, the system $\left\{r \mathbf{B}, A_{i}: i \in I\right\}$ is linearly regular.

Proof. Write $A=\bigcap_{i \in I} A_{i}$ and let $r>0$. By assumption, there exists $k_{r}>0$ such that

$$
d_{A}(x) \leq k_{r} \sup _{i \in I} d_{A_{i}}(x) \quad \text { for each } x \in r \mathbf{B} .
$$

Let $f$ be defined by $f(x):=k_{r} \sup _{i \in J} d_{A_{i}}(x)-d_{A}(x)$ for each $x \in X$. From the (4.28), we see that $f(x) \geq 0$ for all $x \in r \mathbf{B}$, and the equality holds for all $x \in \bigcap_{i \in I} A_{i} \cap r \mathbf{B}$. Since $f$ is clearly Lipschitz with modulus $k_{r}+1$, it follows from [8, Proposition 2.4.3] that $f(x)+\left(k_{r}+1\right) d_{r \mathbf{B}}(x) \geq 0$ for all $x \in X$. This implies

$$
d_{A}(x) \leq\left(2 k_{r}+1\right) \max \left\{d_{r \mathbf{B}}(x), \sup _{i \in I} d_{A_{i}}(x)\right\} \quad \text { for each } x \in X .
$$

It follows from Lemma 4.5 that

$$
d_{A \cap r \mathbf{B}}(x) \leq 4 \max \left\{d_{r \mathbf{B}}(x), d_{A}(x)\right\} \leq 4\left(2 k_{r}+1\right) \max \left\{d_{r \mathbf{B}}(x), \sup _{i \in I} d_{A_{i}}(x)\right\} \quad \text { for each } x \in X .
$$

This completes the proof.

For the following corollary, we need to state a lemma, which will also be used in the next section.

Lemma 4.6. Let $\left\{D, C_{i}: i \in I\right\}$ be a family of closed convex sets with nonempty intersection. Let $A$ be a closed subset of $X$ such that

$$
D \cap \bigcap_{i \in I} C_{i} \cap \operatorname{int} A \neq \emptyset
$$

If $\left\{D, C_{i}: i \in I\right\}$ has the strong CHIP, then so does $\left\{D \cap A, C_{i}: i \in I\right\}$. As a partial converse result, if $\left\{D \cap A, C_{i}: i \in I\right\}$ has the strong CHIP at some point $a \in D \cap \bigcap_{i \in I} C_{i} \cap \operatorname{int} A$, so does $\left\{D, C_{i}: i \in I\right\}$. 
Proof. Suppose that $\left\{D, C_{i}: \quad i \in I\right\}$ has the strong CHIP. Also, by (4.30) and a (normed space) generalization [26, Theorem 2.2] of Theorem Deutsch-Li-Ward in [12], $\left\{A, D \cap \bigcap_{i \in I} C_{i}\right\}$ has the strong CHIP. Consequently, for any $x \in D \cap A \cap \bigcap_{i \in I} C_{i}$, one has

$$
\begin{aligned}
N_{D \cap A \cap \bigcap_{i \in I} C_{i}}(x) & \subseteq N_{A}(x)+N_{D \cap \bigcap_{i \in I} C_{i}}(x) \\
& \subseteq N_{A}(x)+N_{D}(x)+\sum_{i \in I} N_{C_{i}}(x) \\
& \subseteq N_{D \cap A}(x)+\sum_{i \in I} N_{C_{i}}(x),
\end{aligned}
$$

which shows that $\left\{D \cap A, C_{i}: i \in I\right\}$ has the strong CHIP.

Conversely, suppose that $a \in D \cap \bigcap_{i \in I} C_{i} \cap \operatorname{int} A$ and that

$$
N_{D \cap A \cap \bigcap_{i \in I} C_{i}}(a) \subseteq N_{D \cap A}(a)+\sum_{i \in I} N_{C_{i}}(a) .
$$

It follows that

$$
N_{D \cap \bigcap_{j \in J} C_{j}}(a) \subseteq N_{D \cap A}(a)+\sum_{j \in J} N_{C_{j}}(a)=N_{D}(a)+N_{A}(a)+\sum_{j \in J} N_{C_{j}}(a)=N_{D}(a)+\sum_{j \in J} N_{C_{j}}(a)
$$

as $N_{A \cap D}(a)=N_{D}(a)+N_{A}(a)=N_{D}(a)$, thanks to [26, Theorem 2.2].

Corollary 4.1. Suppose that $i \mapsto C_{i}$ is lower semicontinuous on $I$ and that either $I$ is finite or there exists an index $i_{0} \in I$ such that $\operatorname{dim} C_{i_{0}}<+\infty$. If $\left\{C_{i}: i \in I\right\}$ is boundedly linearly regular, then it has the strong CHIP.

Proof. Fix any $x \in \bigcap_{i \in I} C_{i}$. Let $r=\|x\|+1$. Since $\left\{C_{i}: i \in I\right\}$ is boundedly linearly regular, we obtain from Proposition 4.1 that $\left\{r \mathbf{B}, C_{i}: i \in I\right\}$ is linearly regular. Taking an index $i_{\infty} \notin I$, set $I_{\infty}=I \cup\left\{i_{\infty}\right\}$ and $C_{i_{\infty}}=r \mathbf{B}$. Clearly the map $i \mapsto C_{i}$ is lower semcontinuous on $I_{\infty}$. It now follows from the assumptions and Theorem 4.4 that $\left\{C_{i}: i \in I_{\infty}\right\}$ satisfies the SECQ and so does $\left\{r \mathbf{B}, C_{i}: i \in I\right\}$; thus $\left\{r \mathbf{B}, C_{i}: i \in I\right\}$ has the strong CHIP (thanks to Theorem 3.1). Then it follows from Lemma 4.6 (with $D=X$ and $A=r \mathbf{B}$ ) that $\left\{C_{i}: i \in I\right\}$ has the strong CHIP at $x$ because $x \in \operatorname{int} r \mathbf{B}$. The proof is complete.

\section{Interior-point conditions and the SECQ}

Recall that $I$ is an index-set and $C=\bigcap_{i \in I} C_{i} \subseteq X$. As in [26], the family $\left\{D, C_{i}: \quad i \in I\right\}$ is called a closed convex set system with base-set $D$ (CCS-system with base-set $D)$ if $D$ and each $C_{i}$ are closed convex subsets of $X$. Furthermore, throughout the remainder of this section, we always assume that $I$ is a compact metric space and $0 \in D \cap C$. Thus,

$$
\sigma_{D} \text { and } \sigma_{C_{i}} \text { are nonnegative functions on } X^{*} \text { for all } i \in I \text {. }
$$

Let $|J|$ denote the cardinality of the set $J$.

Definition 5.1. Let $\left\{D, C_{i}: i \in I\right\}$ be a CCS-system with base-set $D$. Let $m$ be a positive integer. Then the CCS-system $\left\{D, C_{i}: i \in I\right\}$ is said to satisfy: 
(i) the $m$-D-interior-point condition if, for any subset $J$ of $I$ with $|J| \leq \min \{m,|I|\}$,

$$
D \bigcap\left(\bigcap_{i \in J} \operatorname{rint}_{D} C_{i}\right) \neq \emptyset ;
$$

(ii) the $m$-interior-point condition if, for any subset $J$ of $I$ with $|J| \leq \min \{m,|I|\}$,

$$
D \bigcap\left(\bigcap_{i \in J} \operatorname{int} C_{i}\right) \neq \emptyset .
$$

Before proving our main theorems, we first give the following lemma. Recall that $\left.y^{*}\right|_{Z} \in Z^{*}$ is the restriction to $Z$ of $y^{*}$.

Lemma 5.1. Let $m$ be a positive integer and and let $\left\{D, C_{i}: i \in I\right\}$ be a CCS-system with the base-set $D$ satisfying the following conditions.

(a) $D$ is finite dimensional.

(b) The set-valued mapping $i \mapsto(\operatorname{span} D) \cap C_{i}$ is lower semicontinuous on $I$.

(c) The system $\left\{D, C_{i}: i \in I\right\}$ satisfies $m$-D-interior-point condition.

Let $\left(y^{*}, \alpha\right) \in X^{*} \times \mathbb{R}$ and let $\left\{\left(y_{k}^{*}, \alpha_{k}\right)\right\} \subseteq X^{*} \times \mathbb{R}$ be a sequence such that

$$
\left(\left.y_{k}^{*}\right|_{\operatorname{span} D}, \alpha_{k}\right) \text { converges to }\left(\left.y^{*}\right|_{\operatorname{span} D}, \alpha\right) \text {, }
$$

where each $\left(\left.y_{k}^{*}\right|_{\operatorname{span} D}, \alpha_{k}\right)$ can be expressed in the form

$$
\left(\left.y_{k}^{*}\right|_{\operatorname{span} D}, \alpha_{k}\right)=\left(\left.v_{k}^{*}\right|_{Z}, \beta_{k}\right)+\sum_{j=1}^{m}\left(\left.x_{i_{j}^{k}}^{*}\right|_{\operatorname{span} D}, \alpha_{i_{j}^{k}}\right)
$$

with

$$
\left(v_{k}^{*}, \beta_{k}\right) \in \operatorname{epi} \sigma_{D},\left(x_{i_{j}^{k}}^{*}, \alpha_{i_{j}^{k}}\right) \in \operatorname{epi} \sigma_{C_{i_{j}^{k}}}
$$

for some $i_{1}^{k}, \cdots, i_{m}^{k} \in I$. Then

$$
\left(y^{*}, \alpha\right) \in \operatorname{epi} \sigma_{D}+\sum_{i \in I} \operatorname{epi} \sigma_{(\operatorname{span} D) \cap C_{i}} .
$$

Proof. Since $I$ is compact, by considering subsequences if necessary, we may assume that there exists $i_{j} \in I$ such that $i_{j}^{k} \rightarrow i_{j}$ for each $j=1, \cdots, m$. By assumption (c), there exist $z \in D$ and $\delta^{\prime}>0$ such that

$$
\mathbf{B}\left(z, \delta^{\prime}\right) \cap \operatorname{span} D \subseteq C_{i_{j}} \cap \operatorname{span} D \quad \text { for each } j=1,2, \cdots, m .
$$

Set for convenience $Z:=\operatorname{span} D$ and $B:=\mathbf{B}(z, \delta) \cap Z$, where $\delta=\frac{\delta^{\prime}}{2}$. Then $B$ is compact, thanks to assumption (a). For any $j=1,2, \cdots, m$. We make use of the assumption (b) and apply Proposition 2.4 at the point $t_{0}:=i_{j}$ of the lower semicontinuous function $i \mapsto C_{i} \cap Z$ to conclude from (5.8) that $B \subseteq C_{i_{j}^{k}} \cap Z$ for all large enough $k$. Do this for each $j=1,2, \cdots, m$ and take $k_{0} \in \mathbb{N}$ large enough such that

$$
B \subseteq C_{i_{j}^{k}} \cap Z \text { for each } 1 \leq j \leq m \text { and } k \geq k_{0} .
$$


Note that, for each $1 \leq j \leq m$ and $k \in \mathbb{N}$,

$$
\sigma_{B}\left(x_{i_{j}^{k}}^{*}\right)=\sup _{x \in B}\left\langle x_{i_{j}^{k}}^{*}, x\right\rangle=\sup _{x \in \delta \mathbf{B} \cap Z}\left\langle x_{i_{j}^{k}}^{*}, x\right\rangle+\left\langle x_{i_{j}^{k}}^{*}, z\right\rangle=\delta \| x_{i_{j}^{k}}^{*}|Z| \mid+\left\langle x_{i_{j}^{k}}^{*}, z\right\rangle .
$$

It follows from (5.9) that,

$$
\alpha_{i_{j}^{k}} \geq \sigma_{C_{i_{j}^{k}}}\left(x_{i_{j}^{k}}^{*}\right) \geq \sigma_{C_{i_{j}^{k}} \cap Z}\left(x_{i_{j}^{k}}^{*}\right) \geq \sigma_{B}\left(x_{i_{j}^{k}}^{*}\right)=\delta\left\|x_{i_{j}^{k}}^{*} \mid Z\right\|+\left\langle x_{i_{j}^{k}}^{*}, z\right\rangle,
$$

provided that $k \geq k_{0}$. Moreover, since $z \in D$ and $\left(v_{k}^{*}, \beta_{k}\right) \in \operatorname{epi} \sigma_{D},(5.5)$ establishes that

$$
\alpha_{k}-\left\langle y_{k}^{*}, z\right\rangle=\beta_{k}-\left\langle v_{k}^{*}, z\right\rangle+\sum_{j=1}^{m}\left(\alpha_{i_{j}^{k}}-\left\langle x_{i_{j}^{k}}^{*}, z\right\rangle\right) \geq \sum_{j=1}^{m}\left(\alpha_{i_{j}^{k}}-\left\langle x_{i_{j}^{k}}^{*}, z\right\rangle\right) .
$$

Combining (5.10) and (5.11) yields that

$$
\alpha_{k}-\left\langle y_{k}^{*}, z\right\rangle \geq \sum_{j=1}^{m}\left(\alpha_{i_{j}^{k}}-\left\langle x_{i_{j}^{k}}^{*}, z\right\rangle\right) \geq \sum_{j=1}^{m} \delta\left\|x_{i_{j}^{k}}^{*} \mid z\right\| .
$$

This implies that $\left\{\left.x_{i_{j}^{k}}^{*}\right|_{Z}: k \in \mathbb{N}\right\}$ is bounded for each $1 \leq j \leq m$ thanks to (5.4). Consequently $\left\{\left.v_{k}^{*}\right|_{Z}: k \in \mathbb{N}\right\}$ is bounded as, by (5.5),

$$
\left\|\left.v_{k}^{*}\right|_{Z}\right\|=\left\|\left.y_{k}^{*}\right|_{Z}-\left(\left.\sum_{j=1}^{m} x_{i_{j}^{k}}^{*}\right|_{Z}\right)\right\| \leq\left\|\left.y_{k}^{*}\right|_{Z}\right\|+\sum_{j=1}^{m}\left\|\left.x_{i_{j}^{k}}^{*}\right|_{Z}\right\| .
$$

Since $Z$ is finite-dimensional ( and by passing to subsequences if necessary) we may assume that for each $j=1,2, \ldots, m$, there exist $\tilde{x}_{i_{j}}^{*}$ and $\tilde{v}^{*} \in Z^{*}$ such that

$$
\left.x_{i_{j}^{k}}^{*}\right|_{Z} \rightarrow \tilde{x}_{i_{j}}^{*} \text { and }\left.v_{k}^{*}\right|_{Z} \rightarrow \tilde{v}^{*} \text { as } k \rightarrow \infty .
$$

Now, observe that from (5.11), (5.12) and the nonnegativity of support functions (see (5.1)), we see that $\left\{\alpha_{i_{j}^{k}}\right\}$ and $\left\{\beta_{k}\right\}$ are bounded. Thus we may also assume that, for each $j, \alpha_{i_{j}^{k}} \rightarrow \hat{\alpha}_{i_{j}}$ for some $\hat{\alpha}_{i_{j}} \in \mathbb{R}$ and that $\beta_{k} \rightarrow \hat{\beta}$ for some $\hat{\beta} \in \mathbb{R}$. Then, by (5.4) and (5.5),

$$
\left.y^{*}\right|_{Z}=\tilde{v}^{*}+\sum_{j=1}^{m} \tilde{x}_{i_{j}}^{*} \quad \text { and } \quad \alpha=\hat{\beta}+\sum_{j=1}^{m} \hat{\alpha}_{i_{j}} .
$$

Let $x_{i_{j}}^{*} \in X^{*}$ be an extension of $\tilde{x}_{i_{j}}^{*}$ to $X$ and $v^{*} \in X^{*}$ be an extension of $\tilde{v}^{*}$ to $X$. We claim that $\left(x_{i_{j}}^{*}, \hat{\alpha}_{i_{j}}\right) \in$ epi $\sigma_{\left(C_{i_{j}} \cap Z\right)}$. In fact, for $1 \leq j \leq m$, for each $x \in C_{i_{j}} \cap Z$, by assumption (b), there exists a sequence $\left\{x_{i_{j}^{k}}\right\}$ with each $x_{i_{j}^{k}} \in C_{i_{j}^{k}} \cap Z$ such that $x_{i_{j}^{k}} \rightarrow x$ as $k \rightarrow \infty$. It follows from (5.7) and (5.13) that,

$$
\left\langle x_{i_{j}}^{*}, x\right\rangle=\lim _{k \rightarrow \infty}\left\langle x_{i_{j}^{k}}^{*}, x_{i_{j}^{k}}\right\rangle \leq \lim _{k \rightarrow \infty} \alpha_{i_{j}^{k}}=\hat{\alpha}_{i_{j}} .
$$

Therefore $\sup _{x \in C_{i_{j}} \cap Z}\left\langle x_{i_{j}}^{*}, x\right\rangle \leq \hat{\alpha}_{i_{j}}$ and so $\left(x_{i_{j}}^{*}, \alpha_{i_{j}}\right) \in \operatorname{epi} \sigma_{\left(C_{i_{j}} \cap Z\right)}$. Similarly one can show that,

$$
\left\langle v^{*}, x\right\rangle=\lim _{k \rightarrow \infty}\left\langle v_{k}^{*}, x\right\rangle \leq \lim _{k \rightarrow \infty} \beta_{k}=\hat{\beta} \quad \text { for each } x \in D
$$

that is $\left(v^{*}, \hat{\beta}\right) \in$ epi $\sigma_{D}$. Write $\hat{y}^{*}=y^{*}-v^{*}-\sum_{j=1}^{m} x_{i_{j}}^{*}$. Then by (5.14), $\hat{y}^{*} \in Z^{\perp}$ and

$$
\left(y^{*}, \alpha\right)=\left(\hat{y}^{*}, 0\right)+\left(v^{*}, \hat{\beta}\right)+\sum_{j=1}^{m}\left(x_{i_{j}}^{*}, \hat{\alpha}_{i_{j}}\right) \in Z^{\perp} \times\{0\}+\text { epi } \sigma_{D}+\sum_{j=1}^{m} \operatorname{epi} \sigma_{C_{i_{j}} \cap Z} .
$$

Thus, (5.7) holds as $Z^{\perp} \times\{0\}$ is clearly contained in epi $\sigma_{D}$. 
Remark 5.1. If, for (a) of Lemma 5.1, $\operatorname{dim} D \leq m-1$, then the following implication is valid:

$$
(\mathbf{a})+(\mathbf{b})+(\mathbf{c}) \Rightarrow\left\{D,(\operatorname{span} D) \cap C_{i}: i \in I\right\} \text { satisfies the SECQ. }
$$

(This can be seen from (i) of Theorem 5.1 below, but with $m$ replaced by $m-1$.)

Theorem 5.1. Let $m \in \mathbb{N}$ and let $\left\{D, C_{i}: i \in I\right\}$ be a CCS-system with the base-set $D$. We consider the following conditions.

(a) $D$ is of finite dimension $m$.

(b) The set-valued mapping $i \mapsto(\operatorname{span} D) \cap C_{i}$ is lower semicontinuous on $I$.

(c) The system $\left\{D, C_{i}: i \in I\right\}$ satisfies $(m+1)$-D-interior-point condition.

(d) For each $i \in I$, the pair $\left\{D, C_{i}\right\}$ has the property:

$$
\text { epi } \sigma_{(\operatorname{span} D) \cap C_{i}} \subseteq \operatorname{epi} \sigma_{D}+\operatorname{epi} \sigma_{C_{i}}
$$

(e.g. $\left\{D, C_{i}\right\}$ satisfies the $\left.S E C Q\right)$,

(c*) The system $\left\{D, C_{i}: i \in I\right\}$ satisfies $m$-D-interior-point condition.

(d*) For each finite subset $J$ of $I$ with $|J|=\min \{m+1,|I|\}$, the subsystem $\left\{D, C_{j}: j \in J\right\}$ satisfies the $S E C Q$.

Then the following assertions hold.

(i) If (a), (b), (c) are satisfied, then $\left\{D,(\operatorname{span} D) \cap C_{i}: i \in I\right\}$ satisfies the SECQ.

(ii) If (a), (b), (c), (d) are satisfied, then $\left\{D, C_{i}: i \in I\right\}$ satisfies the SECQ.

(iii) If $D$ is bounded and $(\mathbf{a}),(\mathbf{b}),\left(\mathbf{c}^{*}\right),\left(\mathbf{d}^{*}\right)$ are satisfied, then $\left\{D, C_{i}: i \in I\right\}$ satisfies the SECQ.

Proof. (i) Write $Z:=\operatorname{span} D$ as before. For a subset $H$ of $X^{*} \times \mathbb{R}$, we use $\left.H\right|_{Z} \subseteq Z^{*} \times \mathbb{R}$ to denote the restriction to $Z$ of $H$ defined by

$$
\left.H\right|_{Z}=\left\{\left(\left.x^{*}\right|_{Z}, \beta\right):\left(x^{*}, \beta\right) \in H\right\} .
$$

Let $\left(y^{*}, \alpha\right) \in \overline{\operatorname{epi} \sigma_{D}+\sum_{i \in I} \text { epi } \sigma_{C_{i}}} w^{*}$. Since $Z$ is finite dimensional, there exists a sequence $\left\{\left(y_{k}^{*}, \alpha_{k}\right)\right\} \subseteq$ $X^{*} \times \mathbb{R}$ with

$$
\left(y_{k}^{*}, \alpha_{k}\right) \in \operatorname{epi} \sigma_{D}+\sum_{i \in I} \text { epi } \sigma_{C_{i}} \quad \text { for each } k \in \mathbb{N}
$$

such that $\left(\left.y_{k}^{*}\right|_{Z}, \alpha_{k}\right)$ converges to $\left(\left.y^{*}\right|_{Z}, \alpha\right)$. By (5.18) we express for each $k \in \mathbb{N}$,

$$
\left(y_{k}^{*}, \alpha_{k}\right)=\left(v_{k}^{*}, \beta_{k}\right)+\left(u_{k}^{*}, \gamma_{k}\right) .
$$

where $\left(v_{k}^{*}, \beta_{k}\right) \in$ epi $\sigma_{D}$ and $\left(u_{k}^{*}, \gamma_{k}\right) \in \sum_{i \in I}$ epi $\sigma_{C_{i}}$. Since $\left.\left(\sum_{i \in I}\right.$ epi $\left.\sigma_{C_{i}}\right)\right|_{Z}$ is a convex cone in the $(m+1)$-dimensional space $Z^{*} \times \mathbb{R}$, it follows from [30, Theorem 3.15] that, for each $k$, there exist indices $\left\{i_{1}^{k}, \cdots, i_{m+1}^{k}\right\} \subseteq I$ and $\left\{\left(x_{i_{1}^{k}}^{*}, \alpha_{i_{1}^{k}}\right), \cdots,\left(x_{i_{m+1}^{k}}^{*}, \alpha_{i_{m+1}^{k}}\right)\right\}$ with $\left(x_{i_{j}^{k}}^{*}, \alpha_{i_{j}^{k}}\right) \in$ epi $\sigma_{C_{i_{j}^{k}}}$ for each $1 \leq j \leq m+1$ such that

$$
\left(\left.u_{k}^{*}\right|_{Z}, \gamma_{k}\right)=\sum_{j=1}^{m+1}\left(\left.x_{i_{j}^{k}}^{*}\right|_{Z}, \alpha_{i_{j}^{k}}\right) \quad \text { for each } k \in \mathbb{N} .
$$


Thus we have

$$
\left(\left.y_{k}^{*}\right|_{Z}, \alpha_{k}\right)=\left(\left.v_{k}^{*}\right|_{Z}, \beta_{k}\right)+\sum_{j=1}^{m+1}\left(\left.x_{i_{j}^{k}}^{*}\right|_{Z}, \alpha_{i_{j}^{k}}\right) \quad \text { for each } k \in \mathbb{N}
$$

By Lemma 5.1 and thanks to assumptions (a), (b), (c),

$$
\left(y^{*}, \alpha\right) \in \operatorname{epi} \sigma_{D}+\sum_{i \in I} \operatorname{epi} \sigma_{Z \cap C_{i}} .
$$

We have just proved the inclusion

$$
\overline{\text { epi } \sigma_{D}+\sum_{i \in I} \text { epi } \sigma_{C_{i}}} w^{*} \subseteq \text { epi } \sigma_{D}+\sum_{i \in I} \operatorname{epi} \sigma_{Z \cap C_{i}} \text {. }
$$

Noting $D \cap \bigcap_{i \in I}\left(Z \cap C_{i}\right)=D \cap \bigcap_{i \in I} C_{i}$, it follows from (2.15) and (5.23) that

$$
\text { epi } \sigma_{D \cap \bigcap_{i \in I}\left(Z \cap C_{i}\right)}=\overline{\text { epi } \sigma_{D}+\sum_{i \in I} \operatorname{epi} \sigma_{C_{i}}} w^{*} \subseteq \text { epi } \sigma_{D}+\sum_{i \in I} \text { epi } \sigma_{Z \cap C_{i}} .
$$

Thus $\left\{D,(\operatorname{span} D) \cap C_{i}: i \in I\right\}$ satisfies the SECQ by Corollary 2.1. This proves assertion (i).

(ii) Now suppose in addition that (d) is also satisfied. Then (5.24) implies that

$$
\text { epi } \sigma_{D \cap \bigcap_{i \in I} C_{i}} \subseteq \text { epi } \sigma_{D}+\sum_{i \in I}\left(\text { epi } \sigma_{D}+\text { epi } \sigma_{C_{i}}\right) \subseteq \text { epi } \sigma_{D}+\sum_{i \in I} \text { epi } \sigma_{C_{i}} .
$$

By Corollary 2.1 again, this implies that $\left\{D, C_{i}: i \in I\right\}$ satisfies the SECQ, that is, (ii) holds.

(iii) Now suppose that $(\mathbf{a}),(\mathbf{b}),\left(\mathbf{c}^{*}\right),\left(\mathbf{d}^{*}\right)$ are satisfied. Without loss of generality, we may assume that $m+1 \leq|I|$ since, otherwise, the conclusion follows from assumption $\left(\mathbf{d}^{*}\right)$. Consider $\left(y^{*}, \alpha\right),\left(y_{k}^{*}, \alpha_{k}\right),\left(v_{k}^{*}, \beta_{k}\right),\left(u_{k}^{*}, \gamma_{k}\right)$ satisfying (5.18)-(5.21). Let $k \in \mathbb{N}$ and set $I^{k}=\left\{i_{1}^{k}, \cdots, i_{m+1}^{k}\right\}$. Then for any $z \in D \cap \bigcap_{j \in I^{k}} C_{j}(\subseteq Z)$,

$$
\alpha_{k}=\beta_{k}+\sum_{j \in I^{k}} \alpha_{i_{j}^{k}} \geq \sigma_{D}\left(v_{k}^{*}\right)+\sum_{j=1}^{m+1} \sigma_{C_{j}}\left(x_{i_{j}^{k}}^{*}\right) \geq\left\langle v_{k}^{*}+\sum_{j=1}^{m+1} x_{i_{j}^{k}}^{*}, z\right\rangle=\left\langle y_{k}^{*}, z\right\rangle,
$$

thanks to (5.21). Since $D \cap\left(\bigcap_{j \in I^{k}} C_{j}\right)$ is compact, there exists $x^{k} \in D \cap\left(\bigcap_{j \in I^{k}} C_{j}\right)$ such that

$$
\alpha_{k} \geq\left\langle y_{k}^{*}, x^{k}\right\rangle=\sigma_{D \cap\left(\cap_{j \in I^{k}} C_{j}\right)}\left(y_{k}^{*}\right),
$$

i.e., $y_{k}^{*} \in N_{D \cap\left(\bigcap_{j \in I^{k}} C_{j}\right)}\left(x^{k}\right)$. It follows from assumption $\left(\mathbf{d}^{*}\right)$ and Theorem 3.1 that $y_{k}^{*} \in N_{D}\left(x^{k}\right)+$ $\sum_{j \in I^{k}} N_{C_{j}}\left(x^{k}\right)$. Applying [30, Theorem 3.15] to the $m$-dimensional subspace $Z,\left.y_{k}^{*}\right|_{Z}$ can be expressed in the form

$$
\left.y_{k}^{*}\right|_{Z}=\left.d_{k}^{*}\right|_{Z}+\left.\sum_{j \in J^{k}} z_{j}^{*}\right|_{Z},
$$

for some $d_{k}^{*} \in N_{D}\left(x^{k}\right)$ and $z_{j}^{*} \in N_{C_{j}}\left(x^{k}\right)\left(j \in J^{k}\right)$, where $J^{k}$ is subset of $I^{k}$ with $m$ element. Evaluating (5.27) at $x^{k} \in D \cap\left(\bigcap_{j \in I^{k}} C_{j}\right)$, and invoking (2.5) and (5.26), we have

$$
\alpha_{k} \geq\left\langle y_{k}^{*}, x^{k}\right\rangle=\sigma_{D}\left(d_{k}^{*}\right)+\sum_{j \in J^{k}} \sigma_{C_{j}}\left(z_{j}^{*}\right)
$$


Define

$$
\mu_{k}=\alpha_{k}-\sum_{j \in J^{k}} \sigma_{C_{j}}\left(z_{j}^{*}\right)
$$

Then $\mu_{k} \geq \sigma_{D}\left(d_{k}^{*}\right)$ by (5.28). Denoting $\sigma_{C_{j}}\left(z_{j}^{*}\right)$ by $\gamma_{j}$, this and (5.27) imply that

$$
\left(\left.y_{k}^{*}\right|_{Z}, \alpha_{k}\right)=\left(\left.d_{k}^{*}\right|_{Z}, \mu_{k}\right)+\sum_{j \in J^{k}}\left(\left.z_{j}^{*}\right|_{Z}, \gamma_{j}\right) \text {. }
$$

Noting that $\left(d_{k}^{*}, \mu_{k}\right) \in$ epi $\sigma_{D}$ and $\left(z_{j}^{*}, \gamma_{j}\right) \in$ epi $\sigma_{C_{j}}$ for each $j \in J^{k}$. Since $\left|J^{k}\right|=m$ and thanks to assumptions (a), (b) and $\left(\mathbf{c}^{*}\right)$, Lemma 5.1 asserts that

$$
\left(y^{*}, \alpha\right) \in \operatorname{epi} \sigma_{D}+\sum_{i \in I} \operatorname{epi} \sigma_{\left(Z \cap C_{i}\right)}
$$

Let $i \in I$ and let $J$ be any subset of $I$ such that $i \in J$ and $|J|=m+1$. Then, by assumption (d*), one has that

$$
\text { epi } \sigma_{\left(Z \cap C_{i}\right)} \subseteq \operatorname{epi} \sigma_{\left(D \cap\left(\bigcap_{j \in J} C_{j}\right)\right)} \subseteq \text { epi } \sigma_{D}+\sum_{j \in J} \text { epi } \sigma_{C_{j}} .
$$

Therefore, by (5.30) and (5.31), $\left(y^{*}, \alpha\right) \in \operatorname{epi} \sigma_{D}+\sum_{i \in I}$ epi $\sigma_{C_{i}}$ and thus epi $\sigma_{D}+\sum_{i \in I}$ epi $\sigma_{C_{i}}$ is weakly* closed in the case when assumptions $(\mathbf{a}),(\mathbf{b}),\left(\mathbf{c}^{*}\right)$ and $\left(\mathbf{d}^{*}\right)$ are satisfied. By Corollary 2.1, this implies $\left\{D, C_{i}: i \in I\right\}$ satisfies the SECQ. The proof is complete.

Corollary 5.1. Let $m \in \mathbb{N}$ and let $\left\{D, C_{i}: i \in I\right\}$ be a CCS-system with the base-set $D$ satisfying the following conditions.

(a) $D$ is of finite dimension $m$.

(b) The set-valued mapping $i \mapsto(\operatorname{span} D) \cap C_{i}$ is lower semicontinuous on $I$.

$\left(\mathbf{c}^{+}\right)$The system $\left\{D, C_{i}: i \in I\right\}$ satisfies $(m+1)$-interior-point condition.

Then $\left\{D, C_{i}: i \in I\right\}$ satisfies the $S E C Q$.

Proof. By Lemma 4.4, $\left(\mathbf{c}^{+}\right)$implies the conditions (d) and (c) of Theorem 5.1. Thus, Theorem 5.1 ii) is applicable.

The following corollary, which is a direct consequence of Theorem 5.1 (i), is an improvement of Theorem 1.1.

Corollary 5.2. Let $\left\{D, C_{i}: i \in I\right\}$ be a CCS-system with the base-set D. Let $m \in \mathbb{N}$ and let $x_{0} \in D \cap C$. Suppose that the following conditions are satisfied.

(a) $D$ is of finite dimension $m$.

(b) The set-valued mapping $i \mapsto(\operatorname{span} D) \cap C_{i}$ is lower semicontinuous on $I$.

(c) The system $\left\{D, C_{i}: i \in I\right\}$ satisfies $(m+1)$-D-interior-point condition.

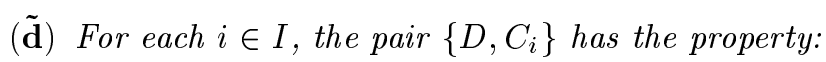

$$
N_{(\operatorname{span} D) \cap C_{i}}\left(x_{0}\right) \subseteq N_{D}\left(x_{0}\right)+N_{C_{i}}\left(x_{0}\right) .
$$


Then the system $\left\{D, C_{i}: i \in I\right\}$ has the strong CHIP at $x_{0}$.

The following corollary is an important improvement of Theorem 1.2. Our main improvement lies in the fact that we need not require the upper semicontinuity of the set valued map $i \mapsto(\operatorname{span} D) \cap C_{i}$ and that (d) can be weakened to required only the subsystems $\left\{D, C_{j}: j \in J\right\}$ with $|J|=l+1$ have the strong CHIP.

Corollary 5.3. Let $m \in \mathbb{N}$ and let $\left\{D, C_{i}: i \in I\right\}$ be a CCS-system with the base-set $D$ satisfying the following conditions.

(a) $D$ is of finite dimension $m$.

(b) The set-valued mapping $i \mapsto(\operatorname{span} D) \cap C_{i}$ is lower semicontinuous on $I$.

(c*) The system $\left\{D, C_{i}: i \in I\right\}$ satisfies $m-D$-interior-point condition.

(d) For each finite subset $J$ of $I$ with $|J|=\min \{m+1,|I|\}$, the subsystem $\left\{D, C_{j}: j \in J\right\}$ has the strong CHIP.

Then the system $\left\{D, C_{i}: i \in I\right\}$ has the strong CHIP.

Proof. If $|I|<m+1$, then $\min \{m+1,|I|\}=|I|$, so the result is trivially true by (d). Thus we may assume that $|I| \geq m+1$. Recall that $C=\bigcap_{i \in I} C_{i}$ and let $x \in D \cap C$. We have to show that the system has the strong CHIP at $x$. For this end, let $\tilde{D}=D \cap \mathbf{B}\left(x, r_{x}\right)$, where $r_{x}=\|x\|+1$. Consider the system $\left\{\tilde{D}, C_{i}: i \in I\right\}$. We claim that the following conditions hold.

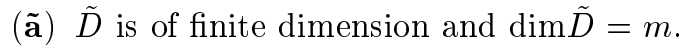

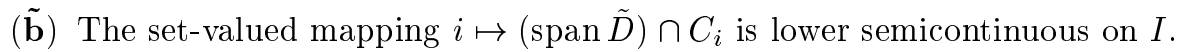

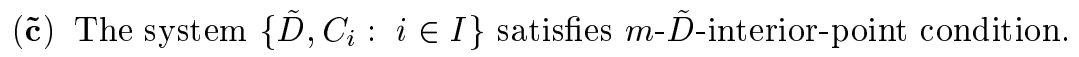

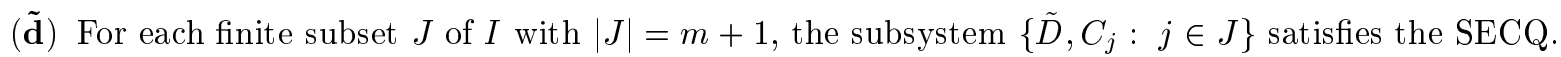

In fact, by assumption ( $\left.\mathbf{c}^{*}\right)$, for each finite subset $J$ of $I$ with $|J|=m$, there exist $\bar{x} \in D$ and $\delta>0$ such that $\mathbf{B}(\bar{x}, \delta) \cap \operatorname{span} D \subseteq D \cap\left(\bigcap_{j \in J} C_{j}\right)$. Since $0 \in \operatorname{int} \mathbf{B}\left(x, r_{x}\right)$, there exists $\lambda \in(0,1)$ such that $\lambda \mathbf{B}(\bar{x}, \delta) \subseteq \mathbf{B}\left(x, r_{x}\right)$. Consequently,

$$
\lambda \mathbf{B}(\bar{x}, \delta) \bigcap \operatorname{span} D \subseteq \lambda D \bigcap \mathbf{B}\left(x, r_{x}\right) \bigcap\left(\bigcap_{j \in J} C_{j}\right) \subseteq \tilde{D} \bigcap\left(\bigcap_{i \in J} C_{i}\right) .
$$

This implies that int $\mathbf{B}(\bar{x}, \delta) \cap$ ri $D \neq \emptyset$; hence

$$
\operatorname{span} \tilde{D}=\operatorname{span} D \text {. }
$$

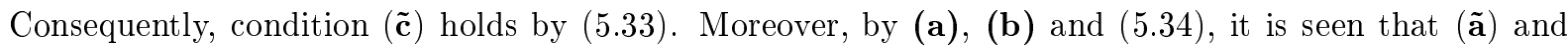

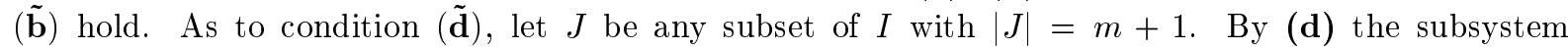
$\left\{D, C_{j}: j \in J\right\}$ has the strong CHIP. Since $x \in \operatorname{int} \mathbf{B}\left(x, r_{x}\right) \cap\left(D \cap\left(\bigcap_{j \in J} C_{j}\right)\right)$, and applying Lemma 4.6 to the ball with center $x$, radius $r_{x}$ and $J$ in place of $A$ and $I$, it follows that $\left\{\tilde{D}, C_{j}: j \in J\right\}$ has the strong CHIP and consequently satisfies the SECQ, thanks to Corollary 3.1 (i) because $\tilde{D} \cap\left(\bigcap_{j \in J} C_{j}\right)$ is compact. Thus $(\tilde{\mathbf{d}})$ is established. Thus Part (iii) of Theorem 5.1 is applicable to concluding that the system $\left\{\tilde{D}, C_{i}: i \in I\right\}$ satisfies the SECQ, which in turn implies that it has the strong CHIP at $x$. Consequently, the system has the strong CHIP at $x$ by Lemma 4.6 applied to the ball with center $x$, radius $r_{x}$ and $J$ in place of $A$ and $I$. The proof is complete. 


\section{References}

[1] J. P. Aubin and H. Frankowska, Set-valued Analysis, Birkhäuser, Boston, 1990.

[2] A. Auslender and M. Teboulle, Asymptotic Cones and Functions in Optimization and Variational Inequalities, Springer-Verlag, New York, Monographs in Mathematics, 2003.

[3] A. Baken, F. Deutsch and W. Li, Strong CHIP, normality, and linear regularity of convex sets, Trans. Amer. Math. Soc., 357 (2005), pp. 3831-3863.

[4] H. Bauschke, Projection Algorithms and Monotone Operators, PhD. Thesis, Simon Fraser University, Depatment of Mathematics, Burnaby, British Columbia V5A 1S6, Canada, August 1996. Available at http://www.cecm.sfu.ca/preprints/1996pp.html

[5] H. Bauschke and J. Borwein, On Projection algorithms for solving convex feasibility problems, SIAM Rev. 38(1996), pp. 367-426.

[6] H. Bauschke, J. Borwein and W. Li, Strong conical hull intersection property, bounded linear regularity, Jameson's property $(G)$, and error bounds in convex optimization, Math. Program., Ser A, 86(1999), pp. $135-160$.

[7] R. S. Burachik and V. Jeyakumar, A simple closure condition for the normal cone intersection formula, Proc. Amer. Math. Soc. 133, 6 (2005), pp. 1741-1748.

[8] F. Clarke, Optimization and Nonsmooth Analysis, John Wiley \& Sons, Inc., New York, 1983.

[9] F. Deutsch, The role of the strong conical hull intersection property in convex optimization and approximation, in Approximation Theory IX, Vol. I: Theoretical Aspects, C. Chui and L. Schumaker, eds., Vanderbilt University Press, Nashville, TN, 1998, pp. 105-112.

[10] F. Deutsch, Best Approximation in Inner Product Spaces, Springer, New York, 2001.

[11] F. Deutsch, W. Li and J. Swetits, Fenchel duality and the strong conical hull intersection property, J. Optim. Theory Appl., 102 (1997), pp. 681-695.

[12] F. Deutsch, W. Li and J. Ward, A dual approach to constrained interpolation from a convex subset of Hilbert space, J. Approx. Theory, 90 (1997), pp. 385-414.

[13] F. Deutsch, W. Li and J. Ward, Best approximation from the intersection of a closed convex set and a polyhedron in Hilbert space, weak Slater conditions, and the strong conical hull intersection property, SIAM J. Optim., 10 (1999), pp. 252-268.

[14] J. Hiriart-Urruty and C. Lemarechal, Convex Analysis and Minimization Algorithms I, Vol. 305 of Grundlehren der Mathematschen Wissenschaften, Springer, New York, 1993.

[15] J. Hiriart-Urruty and C. Lemarechal, Convex Analysis and Minimization Algorithms II, Vol. 306 of Grundlehren der Mathematschen Wissenschaften, Springer, New York, 1993.

[16] G. J. O. Jameson, The duality of pairs of wedges, Proc. Lond. Math. Soc. 24 (1972), pp. 531-547.

[17] V. Jeyakumar, N. Dinh and G. M. Lee, New sequential Lagrange multiplier conditions characterizing optimality without constraint qualification for convex programs, SIAM J.Optim. Vol 14, 2 (2003), pp. 534-547. 
[18] V. Jeyakumar, N. Dinh and G. M. Lee, A new closed cone constraint qualification for convex optimization, Applied Mathematics Research Report AMR 04/6, University of New South Wales.

[19] V. Jeyakumar and H. Mohebi, A global approach to nonlinearly constrained best approximation, Numer. Funct. Anal. Optim., 26(2005), pp. 205-227.

[20] V. Jeyakumar, A. M. Rubinov, B. M. Glover and Y. Ishizuka, Inequality systems and global optimization, J. Mmath. Anal. Appl., 202 (1996), pp. 900-919.

[21] V. Jeyakumar and A. Zaffaroni, Asymptotic conditions for weak and proper optimality in infinite dimensional convex vector optimization, Numer. Funct. Anal. Optim., 17 (1996), pp. 323-343.

[22] A. Lewis and J. S. Pang, Error bounds for convex inequality systems, in Generalized Convexity, Generalized Monotonicity: Recent Results, Proceedings of the Fifth Symposium on Generalized Convexity, Luminy, June 1996, J.-P. Crouzeix, J.-E. Martinez- Legaz, and M.Volle, eds., Kluwer Academic Publishers, Dordrecht, The Netherlands, 1997, pp. 75-110.

[23] C. Li and X. Q. Jin, Nonlinearly constrained best approximation in Hilbert spaces, the strong conical hull intersection property and the basic constraints qualification condition, SIAM J. Optim., 13(2002), pp. 228-239.

[24] C. Li and K. F. Ng, On best approximation by nonconvex sets and perturbation of nonconvex inequality systems in Hilbert spaces, SIAM J. Optim., 13(2002), pp. 726-744.

[25] C. Li and K. F. Ng, Constraint qualification, the strong CHIP and best approximation with convex constraints in Banach spaces, SIAM J. Optim., 14(2003), pp. 584-607.

[26] C. Li and K. F. Ng, Strong CHIP for infinite system of closed convex sets in normed linear spaces, SIAM J.Optim., 16(2005), pp. 311-340.

[27] C. Li and K. F. Ng, On best restricted range approximation in continuous complex-valued fonction spaces, J Approx. Theory, 136(2005), pp. 159-181.

[28] K. F. Ng and W. H. Yang, Regularities and their relationa to error bounds, Math. Program., Ser. A, 99(2004), pp. 521-538.

[29] J. S. Pang, Error bounds in mathematical programming, Math. Program., 79, pp. 299-332 (1997).

[30] R. T. Rockafellar and J. B. Wets, Variational Analysis, Springer-Verlag, New York, 1998.

[31] I. Singer, Duality for optimization and best approximation over finite intersection, Numer. Funct. Anal. Optim., 19(1998), pp. 903-915.

[32] W. Song and R. Zang, Bounded linear regularity of convex sets in Banach spaces and its applications, Math. Program., Ser. A, 106(2006), pp. 59-79.

[33] T. Ströberg, The operation of infimal convolution, Diss. Math., 352 (1996), pp. 1-61.

[34] C. Zălinescu, Convex Analysis in General Vector Spaces, World Scientific Pub., 2002. 